\title{
On generating the set of nondominated solutions of a linear programming problem with parameterized fuzzy numbers
}

\author{
Manuel Arana Jiménez ${ }^{1}$ (D) Carmen Sánchez Gil ${ }^{1}$ \\ Received: 29 November 2018 / Accepted: 4 October 2019 \\ (c) Springer Science+Business Media, LLC, part of Springer Nature 2019
}

\begin{abstract}
The paper presents a new method for solving fully fuzzy linear programming problems with inequality constraints and parameterized fuzzy numbers, by means of solving multiobjective linear programming problems. The equivalence is proven between the set of nondominated solutions of the fully fuzzy linear programming problem and the set of weakly efficient solutions of the considered and related multiobjective linear problem. The whole set of nondominated solutions for a fully fuzzy linear programming problem is explicitly obtained by means of a finite generator set.
\end{abstract}

Keywords Fully fuzzy linear programming problem · Parameterized fuzzy numbers · Multiobjective optimization

\section{Introduction}

Decision making in a fuzzy environment introduced by Bellman and Zadeh [7] is well-known nowadays, and it has been adopted by researchers in fields close to fuzzy linear programming $[10,15,18,21,34,35,45]$. Following the previous referred works, we can see that it was usual that not all parts of the fuzzy linear problem were assumed to be fuzzy. In the study of solutions for fuzzy linear programming problems where all the parameters and variables are fuzzy numbers, let us recall that recently Lofti et al. [33] pointed out that there was no method in the literature. These authors study fully fuzzy linear programming (FFLP) problems and propose a new method to find the fuzzy optimal solution of (FFLP) problems with equality constraints with symmetric fuzzy numbers. In a similar manner, and extending the previous work, Kumar et al. [30] claim that there was no method in the literature to obtain an exact solution of (FFLP) problems with equality constraints, and that in [33] the solutions are approximate, not exact and also it is tough to apply the existing method for finding them. In this regard, they propose a new method for finding the fuzzy optimal solution of (FFLP)

Manuel Arana Jiménez manuel.arana@uca.es

Carmen Sánchez Gil

mcarmen.sanchez@uca.es

1 Department of Statistics and Operational Research, University of Cádiz, Cádiz, Spain 
problems with equality constraints, with triangular fuzzy numbers involved, although they use ranking function (see [3] and the bibliography therein) to compare the objective function values. Najafi and Edalatpanah [39] corrected this method. Khan et al. [27] deal with (FFLP) with inequalities, and they also compare the objective function values via ranking functions (see also [9,28,40]). Ezzati et al. [19] recovered the methods provided by Lofti et al. [33] and Kumar et al. [30] in (FFLP) to propose a new method based on a multiobjective programming problem with equality constraints. To this purpose, they present a new relationship between two triangular fuzzy numbers in order to define an exact optimal solution of (FFLP). This relationship is introduced in terms of the global optimal solution of (FFLP) more than in terms of nondominated solution, and it is not equivalent to that given in [30]. To get an exact optimal solution of (FFLP), the authors propose a lexicographic method. Das et al. [14] apply a lexicographic method with trapezoidal fuzzy numbers.

Taking into account the previous background, Liu and Gao [32] remarked some limitations of the existing methods. As an application to fuzzy transportation problems, we refer to Chakraborty et al. [11], who have updated and applied methods for finding a fuzzy optimal solution.

Recently, Arana-Jiménez [5] has proposed a new method to find the fuzzy optimal (nondominated) solutions of (FFLP) problems with inequality constraints, with triangular fuzzy numbers and not necessarily symmetric, via solving a multiobjective linear problem with crips numbers. To this matter, he proposes an algorithm that does not use ranking functions.

On the other hand, Stafanini et al. [43] (see bibliography therein) have discussed the interest in maintaining the simplicity of computations by the use of simple local monotonic approximations of the lower and upper branches of fuzzy numbers. In this regard, they use approximations to fuzzy numbers through parameterization, using a uniform subdivision of the interval $[0,1]$ to get a finite number of $\alpha$-cuts. Following the idea of approximation and simplicity, Hanss [25] deal with the notion of discretized fuzzy numbers, as well as the decomposed fuzzy numbers, which reduces elementary fuzzy arithmetic to the wellestablished discipline of interval arithmetic, as introduced by Moore [37]. Coroianu et al. [13] illustrate the potential of the piece-wise linear approximation of fuzzy numbers. Recently, Stefanini and Bede [44] propose the LU-parametric representation of a fuzzy number, based on the use of piece-wise differentiable functions, such that few parameters be sufficient to represent or to approximate a fuzzy number. Báez et al. [6], study the polygonal fuzzy numbers, which they consider as a particular case of the parametric representation of fuzzy numbers with linear interpolation. Although there is not a general method for fuzzyfication, which is a subjective assessment and depends on the available information, polygonal fuzzy numbers fit with many types of information or can become a suitable approximation. To this regard, Kávařová and Viertl [29] and Möller et al. [36] provide some methods and examples. As an application of polygonal fuzzy numbers, recently, Shyi et al. [12] propose a new transformation-based weighted fuzzy interpolative reasoning method.

As an extension of the work by Arana-Jiménez [5], and considering polygonal fuzzy numbers as a parameterization of fuzzy numbers, we study a fully fuzzy linear programming (FFLP) problems. To this aim, it is presented a new method to find the fuzzy optimal (nondominated) solutions of (FFLP) problems with inequality constraints, where no ranking functions are needed. We prove an equivalence between the set of the considered fully fuzzy optimal (nondominated) solutions of (FFLP) and the set of weakly efficient solutions of its related multiobjective linear problem. We establish an algorithm to determine the whole set of the nondominated solutions for (FFLP) problem through a finite generator set, based on $[20,46]$. In this manner, a decision-maker gets a set of fuzzy optimal solutions. Since the decision-maker may need a precise quantity for each variable in these fuzzy solutions, there 
are some existing methods to convert fuzzy numbers into crip numbers (see Ross [41], for instance). Although it is not the aim of the present work, we have included some of these methods in the examples to illustrate our results.

\section{Notation and arithmetic on fuzzy numbers}

We denote by $\mathcal{K}_{C}$ the family of all bounded closed intervals in $\mathbb{R}$, i.e.,

$$
\mathcal{K}_{C}=\{[\underline{a}, \bar{a}] \mid \underline{a}, \bar{a} \in \mathbb{R} \text { and } \underline{a} \leq \bar{a}\} .
$$

A fuzzy set on $\mathbb{R}^{n}$ is a mapping $u: \mathbb{R}^{n} \rightarrow[0,1]$. For each fuzzy set $u$, we denote its $\alpha$-level set as $[u]^{\alpha}=\left\{x \in \mathbb{R}^{n} \mid u(x) \geq \alpha\right\}$ for any $\alpha \in(0,1]$. The support of $u$ we denote by $\operatorname{supp}(u)$ where $\operatorname{supp}(u)=\left\{x \in \mathbb{R}^{n} \mid u(x)>0\right\}$. The closure of supp $(u)$ defines the 0 -level of $u$, i.e., $[u]^{0}=\operatorname{cl}(\operatorname{supp}(u))$ where $\operatorname{cl}(M)$ means the closure of the subset $M \subset \mathbb{R}^{n}$. A fuzzy number is a type of fuzzy set (see Dubois an Prade [16,17]), as follows.

Definition 1 A fuzzy set $u$ on $\mathbb{R}$ is said to be a fuzzy number if:

1. $u$ is normal, i.e., there exists $x_{0} \in \mathbb{R}$ such that $u\left(x_{0}\right)=1$;

2. $u$ is an upper semi-continuous function;

3. $u(\lambda x+(1-\lambda) y) \geq \min \{u(x), u(y)\}, x, y \in \mathbb{R}, \lambda \in[0,1]$

4. $[u]^{0}$ is compact.

Let $\mathcal{F}_{C}$ denote the family of all fuzzy numbers. So, for any $u \in \mathcal{F}_{C}$ we have that $[u]^{\alpha} \in \mathcal{K}_{C}$ for all $\alpha \in[0,1]$ and thus the $\alpha$-levels of a fuzzy interval are given by $[u]^{\alpha}=\left[\underline{u}_{\alpha}, \bar{u}_{\alpha}\right]$, $\underline{u}_{\alpha}, \bar{u}_{\alpha} \in \mathbb{R}$ for all $\alpha \in[0,1]$. A fuzzy number $u$ is said to be a non negative fuzzy number if $\underline{u}_{\alpha} \geq 0$, for all $\alpha \in[0,1]$. In [25], we can find the main sets of fuzzy numbers, such as L-R fuzzy numbers, trapezoidal fuzzy numbers, triangular fuzzy numbers, gaussian fuzzy numbers, quasi-gaussian fuzzy numbers, quasi-quadric fuzzy numbers, exponential fuzzy numbers, quasi-exponential fuzzy numbers, and singleton fuzzy numbers. The representation of fuzzy numbers has been deeply discussed by Stefanini et al. [43]. Triangular fuzzy numbers are a particular type of singleton fuzzy numbers, well-known in the literature (see, for instance, $[16,17,26,27,33,43])$ which are well determined and parameterized by three real numbers. So, given $a^{-} \leq a \leq a^{+}$, then a fuzzy number $\tilde{a}=\left(a^{-}, a, a^{+}\right)$is said to be a triangular fuzzy number if its membership function is given by

$$
\tilde{a}(x)=\left\{\begin{array}{lr}
\frac{x-a^{-}}{a-a^{-}}, & \text {if } a^{-} \leq x \leq a, \\
\frac{a^{+}-x}{a^{+}-a}, & \text { if } a<x \leq a^{+} \\
0, & \text { otherwise }
\end{array}\right.
$$

At the same time, given a triangular fuzzy number $\tilde{a}=\left(a^{-}, a, a^{+}\right)$, its $\alpha$-levels are formulated as

$$
[\tilde{a}]^{\alpha}=\left[a^{-}+\left(a-a^{-}\right) \alpha, a^{+}-\left(a^{+}-a\right) \alpha\right]
$$

for all $\alpha \in[0,1]$. The previous formulation of $\alpha$-levels characterizes a unique triangular fuzzy number, what can be established by the connection between a fuzzy number and their endpoint functions (Goestschel and Voxman [23]). 
A fuzzy number $\tilde{a}=\left(a_{0}^{-}, a_{1}^{-}, a_{1}^{+}, a_{0}^{+}\right)$is said to be a trapezoidal fuzzy number if its membership function is given by

$$
\tilde{a}(x)= \begin{cases}\frac{x-a_{0}^{-}}{a_{1}^{-}-a_{0}^{-}}, & \text {if } a_{0}^{-} \leq x<a_{1}^{-} \\ 1, & \text { if } a_{1}^{-} \leq x \leq a_{1}^{+} \\ \frac{a_{0}^{+}-x}{a_{0}^{+}-a_{1}^{+}}, & \text {if } a_{1}^{+}<x \leq a_{0}^{+} \\ 0, & \text { otherwise. }\end{cases}
$$

Its $\alpha$-levels are formulated as

$$
[\tilde{a}]^{\alpha}=\left[a_{0}^{-}+\left(a_{1}^{-}-a_{0}^{-}\right) \alpha, a_{0}^{+}-\left(a_{0}^{+}-a_{1}^{+}\right) \alpha\right],
$$

for all $\alpha \in[0,1]$. Note that a trapezoidal fuzzy number $\tilde{a}$ is triangular if and only if $a_{1}^{-}=$ $a_{1}^{+}$. As an extension of a triangular and trapezoidal fuzzy number, and inspired in other definitions on parametric fuzzy numbers (see, for instance, $[6,25,43,44]$ ), following we review the concept of polygonal fuzzy numbers. Based on the idea that intermediate level sets may be obtained by piecewise linear interpolation of some fixed levels, Báez et al. [6] define the polygonal fuzzy set. This definition, applied to the particular case of fuzzy number, can be formulated as follows. Given $\left\{\alpha_{i}: i=0,1 \ldots, k\right\}$ a partition of the interval [0,1], with $0=\alpha_{0}<\alpha_{1}<, \ldots,<\alpha_{k}=1$, a fuzzy number $\tilde{a}$ is said to be a polygonal fuzzy number if its $\alpha$-levels $[\tilde{a}]^{\alpha}$ satisfies $[\tilde{a}]^{\alpha}=(1-\lambda)[\tilde{a}]^{\alpha_{i}}+\lambda[\tilde{a}]^{\alpha_{i+1}}$, where $0 \leq \alpha_{i}<\alpha \leq \alpha_{i+1} \leq 1$ for some $i=0, \ldots, k-1$ and $\lambda=\lambda(\alpha)=\left(\alpha-\alpha_{i}\right) /\left(\alpha_{i+1}-\alpha_{i}\right)$. The latest means that $\tilde{a}$ has a membership function with polygonal shape (see [6]). Define $[\tilde{a}]^{\alpha_{i}}=\left[a_{i}^{-}, a_{i}^{+}\right]$, for all $i=0,1, \ldots, k$, therefore its membership function is given by

$$
\tilde{a}(x)= \begin{cases}\frac{x-a_{i-1}^{-}}{a_{i}^{-}-a_{i-1}^{-}}\left(\alpha_{i}-\alpha_{i-1}\right)+\alpha_{i-1}, & \text { if } i \in\{1, \ldots, k\} \text { and } a_{i-1}^{-} \leq x<a_{i}^{-}, \\ 1, & \text { if } a_{k}^{-} \leq x \leq a_{k}^{+}, \\ \frac{a_{i-1}^{+}-x}{a_{i-1}^{+}-a_{i}^{+}}\left(\alpha_{i}-\alpha_{i-1}\right)+\alpha_{i-1}, & \text { if } i \in\{1, \ldots, k\} \text { and } a_{i}^{+}<x \leq a_{i-1}^{+}, \\ 0, & \text { otherwise. }\end{cases}
$$

From now on, we refer such fuzzy numbers as $k$-polygonal fuzzy numbers, and denoted as $\tilde{a}=\left(a_{0}^{-}, a_{1}^{-}, \ldots, a_{k}^{-}, a_{k}^{+}, \ldots, a_{1}^{+}, a_{0}^{+}\right)$. In the particular case when $\alpha_{i}=\frac{i}{k}$, then $\tilde{a}$ is just said to be a regular $k$-polygonal fuzzy numbers. And a $k$-polygonal fuzzy number $\tilde{a}$, with respect to $\left\{\alpha_{i}: i=0,1 \ldots, k\right\}$, is said to be non negative when $a_{0}^{-} \geq 0$ (Fig. 1).

Remark 1 Note that given two partitions of [0,1], $P_{1}$ and $P_{2}$, with $P_{1} \subset P_{2}$, then a polygonal fuzzy number with respect to $P_{1}$ is also a polygonal fuzzy number with respect to $P_{2}$. Therefore, if $\tilde{a}$ is a $k$-polygonal fuzzy number with respect to $P_{a}=\left\{\alpha_{i}: i=0,1 \ldots, k\right\}$, and $\tilde{b}$ is a $q$-polygonal fuzzy number with respect to $P_{b}=\left\{\beta_{i}: i=0,1 \ldots, q\right\}$, then $\tilde{a}$ and $\tilde{b}$ are $r$-polygonal fuzzy numbers with respect to $P_{a} \cup P_{b}$, with $r=\operatorname{card}\left(P_{a} \cup P_{b}\right)$, i.e., $r$ is the cardinality of the set $P_{a} \cup P_{b}$. This fact is useful in the sequel when operations are defined between two polygonal fuzzy numbers where, for convenience, the partition will be assumed to be the same for both of them.

In [ [6], Proposition 7], we find a characterization of polygonal fuzzy number respect to a partition $\left\{\alpha_{i}\right\}$ via the family of $\alpha$-levels corresponding to that partition. This result will be useful for the multiplication operation, which we define later (Fig. 2). 


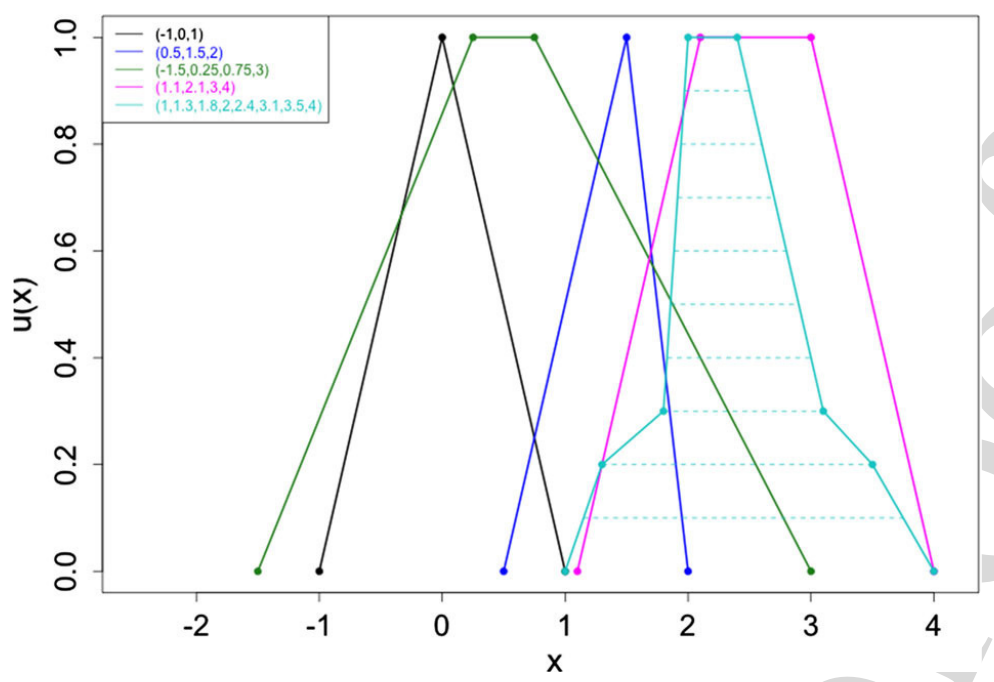

Fig. 1 Example of different Fuzzy numbers: In black and blue-two triangular Fuzzy numbers $\tilde{a}=(-2,0,1)$ and $(0.5,1.5,2)$ With green two trapezoidal Fuzzy numbers $\tilde{b}=(-1.5,0.25,0.75,3)$ and $(1.1,2.1,3,4) . \tilde{a}$ and $\tilde{b}$ have different $x$ range partitions, with the same $[0,1]$ partition. angal fuzzy number $\tilde{c}=(1,1.3,1.8,2,2.4,3.1,3.5,4)$. The $\alpha=0,0.1,0.2, \ldots, 0.9,1$ levels are represented with dashed lines
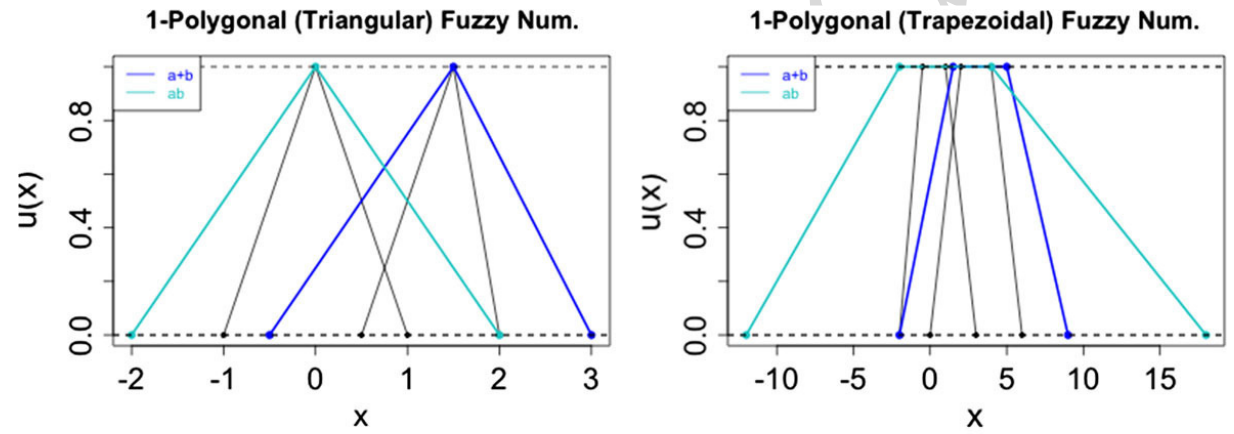

3-Polygonals Fuzzy Num.

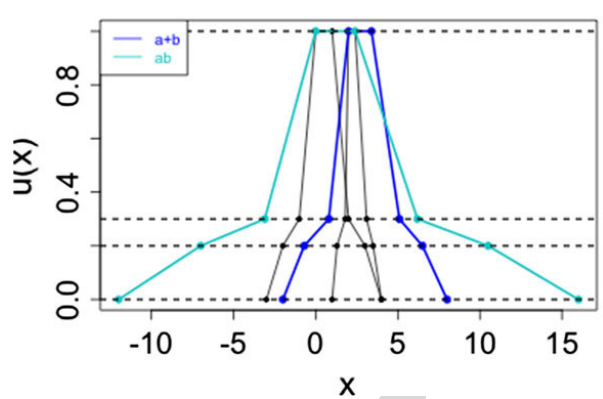

5-Polygonals Fuzzy Num.

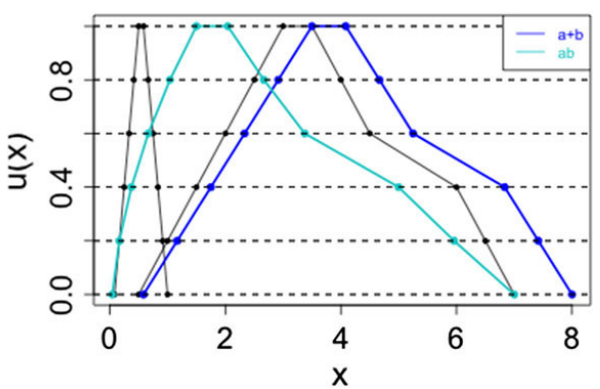

Fig. 2 Fuzzy numbers arithmetics: example of the sum and product of different $k$-polygonal fuzzy numbers, defined in 2

Following, we consider some classical arithmetic operations on interval and fuzzy numbers. Given $A=[\underline{a}, \bar{a}], B=[\underline{b}, \bar{b}] \in \mathcal{K}_{C}$ and $\tau \in \mathbb{R}$ :

$$
\begin{aligned}
& A+B=[\underline{a}+\underline{b}, \bar{a}+\bar{b}], \quad \tau A=\{\tau a: a \in A\}=\left\{\begin{array}{ll}
{[\tau \underline{a}, \tau \bar{a}],} & \text { if } \tau \geq 0, \\
{[\tau \bar{a}, \tau \underline{a}],} & \text { if } \tau \leq 0
\end{array},\right. \\
& A \times B=[\min \{\underline{a b}, \bar{a} \bar{b}, \underline{a} \bar{b}, \bar{a} \underline{b}\}, \max \{\underline{a b}, \bar{a} \bar{b}, \underline{a} \bar{b}, \bar{a} \underline{b}\}] .
\end{aligned}
$$


We refer to Moore [37,38] and Alefeld and Herzberger [1] for further details on the topic of interval analysis. As an extension of interval arithmetic to fuzzy numbers, and referring to $[8,22,31]$, the membership function of the operation $u * v$, with $* \in\{+, \cdot\}$, is defined by

$$
(u * v)(z)=\sup _{z=x * y} \min \{u(x), v(y)\} .
$$

If we consider the fuzzy numbers $u, v$ represented by $\left[\underline{u}_{\alpha}, \bar{u}_{\alpha}\right]$ and $\left[\underline{v}_{\alpha}, \bar{v}_{\alpha}\right]$, respectively, and a real number $\lambda$, then the addition $u+v$, the scalar multiplication $\lambda u$, and the multiplication $u v$ produce fuzzy numbers and can be defined by means of their $\alpha$-levels as follows (see, for instance, [Theorem 2.6, [22]). For any $\alpha \in[0,1]$ :

$$
\begin{aligned}
& {[u+v]^{\alpha}=\left[\underline{u}_{\alpha}+\underline{v}_{\alpha}, \bar{u}_{\alpha}+\bar{v}_{\alpha}\right],} \\
& {[\lambda u]^{\alpha}=\left[\min \left\{\lambda \underline{u}_{\alpha}, \lambda \bar{u}_{\alpha}\right\}, \max \left\{\lambda \underline{u}_{\alpha}, \lambda \bar{u}_{\alpha}\right\}\right],} \\
& {[u v]^{\alpha}=[u]^{\alpha} \times[v]^{\alpha}=\left[\min \left\{\underline{u}_{\alpha} \underline{v}_{\alpha}, \bar{u}_{\alpha} \bar{v}, \underline{u}_{\alpha} \bar{v}_{\alpha}, \bar{u}_{\alpha} \underline{v}_{\alpha}\right\}, \max \left\{\underline{u}_{\alpha} \underline{v}_{\alpha}, \bar{u}_{\alpha} \bar{v}, \underline{u}_{\alpha} \bar{v}_{\alpha}, \bar{u}_{\alpha} \underline{v}_{\alpha}\right\}\right] .}
\end{aligned}
$$

Báez et al. [6] proved that for a fixed partition, the set of polygonal fuzzy numbers with respect to this partition is closed under addition and multiplication by a scalar. However, it is not closed under the multiplication operation. As an example, it is sufficient to consider the well-known case of triangular fuzzy numbers (see, for instance, the examples in [47]). So, to avoid this situation, it is usual to apply a different multiplication operation between triangular fuzzy numbers referenced in $[5,26,27,30]$, among others. The result of this multiplication is a new triangular fuzzy number, which can be considered as an approximation to the multiplication given in (2). Taking into account the previous comments, we propose the following arithmetic operations on the set of polygonal fuzzy numbers.

Definition 2 Given two $k$-polygonal fuzzy numbers $\tilde{a}=\left(a_{0}^{-}, a_{1}^{-}, \ldots, a_{k}^{-}, a_{k}^{+}, \ldots, a_{1}^{+}, a_{0}^{+}\right)$ and $\tilde{b}=\left(b_{0}^{-}, b_{1}^{-}, \ldots, b_{k}^{-}, b_{k}^{+}, \ldots, b_{1}^{+}, b_{0}^{+}\right)$, it is defined the basic arithmetical operations as follows:

(i) The addition $\tilde{a}+\tilde{b}=\tilde{c}$ where $c_{i}^{-}=a_{i}^{-}+b_{i}^{-}$, and $c_{i}^{+}=a_{i}^{+}+b_{i}^{+}$for $i=0,1 \ldots, k$. This is,

$$
\tilde{a}+\tilde{b}=\left(a_{0}^{-}+b_{0}^{-}, a_{1}^{-}+b_{1}^{-}, \ldots, a_{k}^{-}+b_{k}^{-}, a_{k}^{+}+b_{k}^{+}, \ldots, a_{1}^{+}+b_{1}^{+}, a_{0}^{+}+b_{0}^{+}\right)
$$

(ii) The multiplication by a scalar $\lambda \in \mathbb{R}$,

$$
\lambda \tilde{a}= \begin{cases}\left(\lambda a_{0}^{-}, \lambda a_{1}^{-}, \ldots, \lambda a_{k}^{-}, \lambda a_{k}^{+}, \ldots, \lambda a_{1}^{+}, \lambda a_{0}^{+}\right) & \text {if } \lambda \geq 0 ; \\ \left(\lambda a_{0}^{+}, \lambda a_{1}^{+}, \ldots, \lambda a_{k}^{+}, \lambda a_{k}^{-}, \ldots, \lambda a_{1}^{-}, \lambda a_{0}^{-}\right) & \text {if } \lambda<0 .\end{cases}
$$

(iii) The multiplication of two $k$-fuzzy polygonal numbers, $\tilde{a} \tilde{b}=\tilde{c}=\left(c_{0}^{-}, c_{1}^{-}, \ldots, c_{k}^{-}, c_{k}^{+}\right.$, $\left.\ldots, c_{1}^{+}, c_{0}^{+}\right)$, where

$$
\left\{\begin{array}{l}
c_{i}^{-}=\min \left\{a_{i}^{-} b_{i}^{-}, a_{i}^{-} b_{i}^{+}, a_{i}^{+} b_{i}^{-}, a_{i}^{+} b_{i}^{+}\right\} \\
c_{i}^{+}=\max \left\{a_{i}^{-} b_{i}^{-}, a_{i}^{-} b_{i}^{+}, a_{i}^{+} b_{i}^{-}, a_{i}^{+} b_{i}^{+}\right\}
\end{array} \quad i=0,1, \ldots, k .\right.
$$

Proposition 1 For a fixed partition $\left\{\alpha_{i}: i=0,1 \ldots, k\right\}$ of $[0,1]$, the set of $k$-polygonal fuzzy numbers with respect to this partition is closed under addition, multiplication by scalar, and multiplication.

Proof The proof is immediate from Definition 2. 
Remark 2 In Definition 2, operation (i) and (ii) are equivalent to those given in (3) and (4), although this equivalence does not occur between (iii) and (5), even in the particular case that the $k$-polygonal fuzzy numbers reduce to triangular fuzzy numbers, such as we have commented before. However, in the cases that $\alpha=\alpha_{i}, i \in\{0,1, \ldots, k\}$, it follows that $[\tilde{a}]^{\alpha_{i}} \times[\tilde{b}]^{\alpha_{i}}=\left[c_{i}^{-}, c_{i}^{+}\right]$, with $c_{i}^{-}=\min \left\{a_{i}^{-} b_{i}^{-}, a_{i}^{-} b_{i}^{+}, a_{i}^{+} b_{i}^{-}, a_{i}^{+} b_{i}^{+}\right\}$and $c_{i}^{-}=\min \left\{a_{i}^{-} b_{i}^{-}, a_{i}^{-} b_{i}^{+}, a_{i}^{+} b_{i}^{-}, a_{i}^{+} b_{i}^{+}\right\}$. Therefore, the $\alpha$-levels of the multiplication in (iii) and in (5) between two $k$-polygonal fuzzy numbers respect to the same partition, for $\alpha=\alpha_{i}$, $i \in\{0,1, \ldots, k\}$, are equal.

In order to compare two fuzzy numbers, there exist some definitions as generalization of relationship on intervals (see [24]), in the recent literature. In such manner, and recently, Stefanini and Arana-Jiménez [42] have discussed this topic, and proposed a definition on partial order for fuzzy numbers. Based on their definition, we introduce the following. To this regard, given $u, v \in \mathcal{F}_{C}$, we write their $\alpha$-levels as $u_{\alpha}=\left[\underline{u}_{\alpha}, \bar{u}_{\alpha}\right] \in \mathcal{K}_{C}$ and $v_{\alpha}=$ $\left[\underline{v}_{\alpha}, \bar{v}_{\alpha}\right] \in \mathcal{K}_{C}$, respectively, for all $\alpha \in[0,1]$.

Definition 3 Given $u, v \in \mathcal{F}_{C}$, we say that

(i) $u \prec v$ if and only if $\underline{u}_{\alpha}<\underline{v}_{\alpha}$ and $\bar{u}_{\alpha}<\bar{v}_{\alpha}$, for all $\alpha \in[0,1]$.

(ii) $u \leqq v$ if and only if $\underline{u}_{\alpha} \leq \underline{v}_{\alpha}$ and $\bar{u}_{\alpha} \leq \bar{v}_{\alpha}$, for all $\alpha \in[0,1]$.

In a similar way, the relations $\succ$ and $\geqq$ are considered. These relationships provide partial orders in $\mathcal{F}_{C}$. Note that to say $u \leqq v$ and $v \leqq u$ is equivalent to say $u=v$. For convenience, we denote $\tilde{0}$ the fuzzy number whose membership function is valued as 0 at every point. Observe that a polygonal fuzzy number $\tilde{a}$ is nonnegative if and only if $\tilde{a} \geqq \tilde{0}$, that is, $a_{0}^{-} \geq 0$.

Theorem 1 Given two k-polygonal fuzzy numbers $\tilde{a}=\left(a_{0}^{-}, a_{1}^{-}, \ldots, a_{k}^{-}, a_{k}^{+}, \ldots, a_{1}^{+}, a_{0}^{+}\right)$ and $\tilde{b}=\left(b_{0}^{-}, b_{1}^{-}, \ldots, b_{k}^{-}, b_{k}^{+}, \ldots, b_{1}^{+}, b_{0}^{+}\right)$with respect to $\left\{\alpha_{i}: i=0,1 \ldots, k\right\}$, it follows that

(i) $\tilde{a} \prec \tilde{b}$ if and only if $a_{i}^{-}<b_{i}^{-}$, and $a_{i}^{+}<b_{i}^{+}$, for all $i=0,1, \ldots, k$.

(ii) $\tilde{a} \leqq \tilde{b}$ if and only if $a_{i}^{-} \leq b_{i}^{-}$, and $a_{i}^{+} \leq b_{i}^{+}$, for all $i=0,1, \ldots, k$.

Proof To prove the result, firstly let us consider (i), and suppose that $\tilde{a} \prec \tilde{b}$. For the particular case that $\alpha_{i} \in\left\{\alpha_{0}, \alpha_{1}, \ldots, \alpha_{k}\right\}$, it follows that

$$
[\tilde{a}]^{\alpha_{i}}=\left[\underline{\tilde{a}}_{\alpha_{i}}, \overline{\tilde{a}}_{\alpha_{i}}\right]=\left[a_{i}^{-}, a_{i}^{+}\right], \quad[\tilde{b}]^{\alpha_{i}}=\left[\tilde{\tilde{b}}_{\alpha_{i}}, \overline{\tilde{b}}_{\alpha_{i}}\right]=\left[b_{i}^{-}, b_{i}^{+}\right], \quad i=0,1 \ldots, k .
$$

Since $\tilde{a} \prec \tilde{b}$, and by Definition 3, it follows that $\underline{\tilde{u}}_{\alpha}<\underline{\tilde{v}}_{\alpha}$ and $\overline{\tilde{u}}_{\alpha}<\overline{\tilde{v}}_{\alpha}$, for all $\alpha \in[0,1]$; in particular for $\alpha_{i} \in\left\{\alpha_{0}, \alpha_{1}, \ldots, \alpha_{k}\right\}$. In consequence, from (9), $a_{i}^{-}<b_{i}^{-}$, and $a_{i}^{+}<b_{i}^{+}$, for all $i=0,1, \ldots, k$.

Conversely, now let us suppose that $a_{i}^{-}<b_{i}^{-}$, and $a_{i}^{+}<b_{i}^{+}$, for all $i=0,1, \ldots, k$. To prove that $\tilde{a} \prec \tilde{b}$, by Definition 3, let us consider $i \in\{0,1, \ldots, k\}$ and prove that $\underline{\tilde{a}}_{\alpha}<\underline{\tilde{b}}_{\alpha}$ and $\overline{\tilde{a}}_{\alpha}<\overline{\tilde{b}}_{\alpha}$, for all $\alpha \in\left[\alpha_{i-1}, \alpha_{i}\right]$. By hypothesis, it follows that $a_{i-1}^{-}<b_{i-1}^{-}$and $a_{i}^{-}<b_{i}^{-}$. Operating on the previous inequalities, it is derived that

$$
\begin{aligned}
& \frac{\alpha-\alpha_{i-1}}{\alpha_{i}-\alpha_{i-1}} a_{i}^{-}<\frac{\alpha-\alpha_{i-1}}{\alpha_{i}-\alpha_{i-1}} b_{i}^{-}, \\
& \frac{\alpha_{i}-\alpha}{\alpha_{i}-\alpha_{i-1}} a_{i-1}^{-}<\frac{\alpha_{i}-\alpha}{\alpha_{i}-\alpha_{i-1}} b_{i-1}^{-},
\end{aligned}
$$

for all $\alpha \in\left[\alpha_{i-1}, \alpha_{i}\right]$. Combining (10) and (11), we get

$$
\frac{\alpha-\alpha_{i-1}}{\alpha_{i}-\alpha_{i-1}} a_{i}^{-}+\frac{\alpha_{i}-\alpha}{\alpha_{i}-\alpha_{i-1}} a_{i-1}^{-}<\frac{\alpha-\alpha_{i-1}}{\alpha_{i}-\alpha_{i-1}} b_{i}^{-}+\frac{\alpha_{i}-\alpha}{\alpha_{i}-\alpha_{i-1}} b_{i-1}^{-},
$$


which is equivalent to the inequality

$$
\frac{\alpha-\alpha_{i-1}}{\alpha_{i}-\alpha_{i-1}}\left(a_{i}^{-}-a_{i-1}^{-}\right)+a_{i-1}^{-}<\frac{\alpha-\alpha_{i-1}}{\alpha_{i}-\alpha_{i-1}}\left(b_{i}^{-}-b_{i-1}^{-}\right)+b_{i-1}^{-},
$$

for all $\alpha \in\left[\alpha_{i-1}, \alpha_{i}\right]$. Following, consider the hypothesis $a_{i-1}^{+}<b_{i-1}^{+}$and $a_{i}^{+}<b_{i}^{+}$, and proceed as before. Therefore, we get the inequality

$$
a_{i-1}^{+}-\frac{\alpha-\alpha_{i-1}}{\alpha_{i}-\alpha_{i-1}}\left(a_{i-1}^{+}-a_{i}^{+}\right)<b_{i-1}^{+}-\frac{\alpha-\alpha_{i-1}}{\alpha_{i}-\alpha_{i-1}}\left(b_{i-1}^{+}-b_{i}^{+}\right),
$$

for all $\alpha \in\left[\alpha_{i-1}, \alpha_{i}\right]$. Taking into account the expression given by (13) and (14), it is derived that

$$
\begin{aligned}
{[\tilde{a}]^{\alpha} } & =\left[\frac{\alpha-\alpha_{i-1}}{\alpha_{i}-\alpha_{i-1}}\left(a_{i}^{-}-a_{i-1}^{-}\right)+a_{i-1}^{-}, a_{i-1}^{+}-\frac{\alpha-\alpha_{i-1}}{\alpha_{i}-\alpha_{i-1}}\left(a_{i-1}^{+}-a_{i}^{+}\right)\right] \\
& \prec\left[\frac{\alpha-\alpha_{i-1}}{\alpha_{i}-\alpha_{i-1}}\left(b_{i}^{-}-b_{i-1}^{-}\right)+b_{i-1}^{-}, b_{i-1}^{+}-\frac{\alpha-\alpha_{i-1}}{\alpha_{i}-\alpha_{i-1}}\left(b_{i-1}^{+}-b_{i}^{+}\right)\right]=[\tilde{b}]^{\alpha},
\end{aligned}
$$

for all $\alpha \in\left[\alpha_{i-1}, \alpha_{i}\right]$. If we extend the previous reasoning for all $\mathrm{i}=1, \ldots, \mathrm{k}$, then we conclude that $\tilde{a} \prec \tilde{b}$. In a similar manner, (ii) is proved, so the proof is complete.

From the previous theorem, it is easy to derive a similar characterization result for the relationships $\tilde{u} \succ \tilde{v}$ and $\tilde{u} \geqq \tilde{v}$. To illustrate the applicability of the previous result, consider the 2-polygonal fuzzy numbers $\tilde{a}=(1,3,5,7,9,12)$ and $\tilde{b}=(2,3,5,8,9,14)$. It follows that $\tilde{a} \leqq \tilde{b}$, but $\tilde{a} \prec \tilde{b}$ is not verified.

\section{Fully fuzzy linear programming problem}

We consider the following formulation of a Fully Fuzzy Linear Programming Problem:

$$
\begin{aligned}
\text { (FFLP) Min/Max } \tilde{z}=\sum_{n=1}^{N} \tilde{c}_{n} \tilde{x}_{n} \\
\text { subject to } \sum_{n=1}^{N} \tilde{a}_{m n} \tilde{x}_{n} \leqq \tilde{b}_{m}, \quad m=1, \ldots, M \\
\tilde{x}_{n} \geqq 0, \quad n=1, \ldots, N,
\end{aligned}
$$

where $\tilde{z}$ is the fuzzy objective function, $\tilde{c}=\left(\tilde{c}_{1}, \ldots, \tilde{c_{N}}\right)$ is the fuzzy vector with the fuzzy objective function coefficients, $\tilde{x}=\left(\tilde{x}_{1}, \ldots, \tilde{x}_{N}\right)$ is the vector with the $N$ fuzzy decision variables, and $\tilde{a}_{m n}$ and $\tilde{b}_{m}$ are the technical coefficients for the corresponding $M$ constraints of the problem. They are all $k$-polygonal fuzzy numbers with respect to $\left\{0=\alpha_{0}<\alpha_{1}<\right.$ $\left.\cdots<\alpha_{k}=1\right\}$, a partition of the $[0,1]$ interval. Following the established formulation, we have that

$$
\begin{aligned}
\tilde{z} & =\left(z_{0}^{-}, z_{1}^{-}, \ldots, z_{k}^{-}, z_{k}^{+}, \ldots, z_{1}^{+}, z_{0}^{+}\right), \\
\tilde{x}_{n} & =\left(x_{n}^{-}, x_{n}^{-}, \ldots, x_{n_{k}}^{-}, x_{n_{k}}^{+}, \ldots, x_{n_{1}}^{+}, x_{n_{0}}^{+}\right), \quad n=1, \ldots, N, \\
\tilde{c}_{n} & =\left(c_{n_{0}}^{-}, c_{n}^{-}, \ldots, c_{n_{k}}^{-}, c_{n_{k}}^{+}, \ldots, c_{n_{1}^{+}}^{+}, c_{n_{0}}^{+}\right), \quad n=1, \ldots, N, \\
\tilde{a}_{m n} & =\left(a_{m n_{0}}^{-}, a_{m n_{1}}^{-}, \ldots, a_{m n_{k}}^{-}, a_{m n_{k}}^{+}, \ldots, a_{m n_{1}}^{+}, a_{m n_{0}}^{+}\right), \quad m=1, \ldots, M, n=1, \ldots, N, \\
\tilde{b}_{m} & =\left(b_{m_{0}}^{-}, b_{m_{1}}^{-}, \ldots, b_{m_{k}}^{-}, b_{m_{k}}^{+}, \ldots, b_{m_{1}}^{+}, b_{m_{0}}^{+}\right), \quad m=1, \ldots, M .
\end{aligned}
$$


This is, a 2 and 3 -index formulation of the problem. Following the multiplication role given in (8), we have for $n=1, \ldots, N$,

$$
\tilde{c}_{n} \tilde{x}_{n}=\left(\left(\tilde{c}_{n} \tilde{x}_{n}\right)_{0}^{-},\left(\tilde{c}_{n} \tilde{x}_{n}\right)_{1}^{-}, \ldots,\left(\tilde{c}_{n} \tilde{x}_{n}\right)_{k}^{-},\left(\tilde{c}_{n} \tilde{x}_{n}\right)_{k}^{+}, \ldots,\left(\tilde{c}_{n} \tilde{x}_{n}\right)_{1}^{+},\left(\tilde{c}_{n} \tilde{x}_{n}\right)_{0}^{+}\right),
$$

with

$$
\left\{\begin{array}{l}
\left(\tilde{c}_{n} \tilde{x}_{n}\right)_{i}^{-}=\min \left\{c_{n_{i}}^{-} x_{n_{i}}^{-}, c_{n_{i}}^{-} x_{n_{i}}^{+}, c_{n_{i}}^{+} x_{n_{i}}^{-}, c_{n_{i}}^{+} x_{n_{i}}^{+}\right\} \\
\left(\tilde{c}_{n} \tilde{x}_{n}\right)_{i}^{+}=\max \left\{c_{n_{i}}^{-} x_{n_{i}}^{-}, c_{n_{i}}^{-} x_{n_{i}}^{+}, c_{n_{i}}^{+} x_{n_{i}}^{-}, c_{n_{i}}^{+} x_{n_{i}}^{+}\right\}
\end{array} \quad i=0,1, \ldots, k,\right.
$$

and for $n=1, \ldots, N, m=1, \ldots, M$,

$$
\tilde{a}_{m n} \tilde{x}_{n}=\left(\left(\tilde{a}_{m n} \tilde{x}_{n}\right)_{0}^{-}, \ldots,\left(\tilde{a}_{m n} \tilde{x}_{n}\right)_{k}^{-},\left(\tilde{a}_{m n} \tilde{x}_{n}\right)_{k}^{+}, \ldots,\left(\tilde{a}_{m n} \tilde{x}_{n}\right)_{0}^{+}\right),
$$

with

$$
\left\{\begin{array}{l}
\left(\tilde{a}_{m n} \tilde{x}_{n}\right)_{i}^{-}=\min \left\{a_{m n_{i}}^{-} x_{n_{i}}^{-}, a_{m n_{i}}^{-} x_{n_{i}}^{+}, a_{m n_{i}}^{+} x_{n_{i}}^{-}, a_{m n_{i}}^{+} x_{n_{i}}^{+}\right\} \\
\left(\tilde{a}_{m n} \tilde{x}_{n}\right)_{i}^{+}=\max \left\{a_{m n_{i}}^{-} x_{n_{i}}^{-}, a_{m n_{i}}^{-} x_{n_{i}}^{+}, a_{m n_{i}}^{+} x_{n_{i}}^{-}, a_{m n_{i}}^{+} x_{n_{i}}^{+}\right\}
\end{array} \quad i=0,1, \ldots, k .\right.
$$

Remark 3 Since every $\tilde{x}_{n}$ is a nonnegative $k$-polygonal fuzzy number, we have that all expressions for the multiplications above in (FFLP), given in (18) and (19), only depend on the objective coefficients $\tilde{c}_{n}$ and technical coefficients $\tilde{a}_{m n}$ respectively. Therefore, (18) and (19) can be simplified as:

$$
\begin{aligned}
& \left(\tilde{c}_{n} \tilde{x}_{n}\right)_{i}^{-}=\left\{\begin{array}{l}
c_{n_{i}}^{-} x_{n_{i}}^{-} \text {if } c_{n_{i}}^{-} \geq 0 \\
c_{n_{i}}^{-} x_{n_{i}}^{+} \text {other case, }
\end{array} \quad\left(\tilde{a}_{m n} \tilde{x}_{n}\right)_{i}^{-}=\left\{\begin{array}{l}
a_{m n_{i}}^{-} x_{n_{i}}^{-} \text {if } a_{m n_{i}}^{-} \geq 0 \\
a_{m n_{i}}^{-} x_{n_{i}}^{+} \text {other case }
\end{array}\right.\right. \\
& \left(\tilde{c}_{n} \tilde{x}_{n}\right)_{i}^{+}=\left\{\begin{array}{l}
c_{n_{i}}^{+} x_{n_{i}}^{-} \text {if } c_{n_{i}}^{+} \leq 0 \\
c_{n_{i}}^{+} x_{n_{i}}^{+} \text {other case, }
\end{array} \quad\left(\tilde{a}_{m n} \tilde{x}_{n}\right)_{i}^{+}= \begin{cases}a_{m n_{i}}^{+} x_{n_{i}}^{-} & \text {if } a_{m n_{i}}^{+} \leq 0 \\
a_{m n_{i}}^{+} x_{n_{i}}^{+} & \text {other case }\end{cases} \right.
\end{aligned}
$$

We deal with (FFLP) without any kind of ranking function. And, in this regard, we define the following nondominated solution for (FFLP).

Definition 4 Let $\tilde{\bar{x}}$ be a feasible solution for (FFLP). In case of minimization, $\tilde{\bar{x}}$ is said to be a nondominated solution of (FFLP) if there does not exist a feasible solution $\tilde{x}$ for (FFLP) such that $\sum_{n=1}^{N} \tilde{c}_{n} \tilde{x}_{n} \prec \sum_{n=1}^{N} \tilde{c}_{n} \tilde{\bar{x}}_{n}$. And in case of maximization, $\tilde{\bar{x}}$ is said to be a nondominated solution of (FFLP) if there does not exist a feasible solution $\tilde{x}$ for (FFLP) such that $\sum_{n=1}^{N} \tilde{c}_{n} \tilde{x}_{n} \succ \sum_{n=1}^{N} \tilde{c}_{n} \tilde{\bar{x}}_{n}$

Taking into account the previous arithmetic operations, Definition 2, and order relations, Theorem 1, the Fully Fuzzy Linear Programming Problem (FFLP) can be reformulated as a Multiobjective Programming Linear Problem (MOLP). Just developing the previous (FFLP) formulation with the notation described above, we have that 
(MOLP) Min/Max $f(x)=\left(z_{0}^{-}, z_{1}^{-}, \ldots, z_{k}^{-}, z_{k}^{+}, \ldots, z_{1}^{+}, z_{0}^{+}\right)$ subject to $\sum_{n=1}^{N}\left(\tilde{a}_{m n} \tilde{x}_{n}\right)_{i}^{-} \leq b_{m_{i}}^{-}, \quad m=1, \ldots, M, \quad i=0,1, \ldots, k$,

$$
\begin{aligned}
& \sum_{n=1}^{N}\left(\tilde{a}_{m n} \tilde{x}_{n}\right)_{i}^{+} \leq b_{m_{i}}^{+}, \quad m=1, \ldots, M, \quad i=0,1, \ldots, k, \\
& x_{n_{i}}^{-}-x_{n_{j}} \leq 0, \quad 0 \leq i<j \leq k, \quad n=1, \ldots, N, \\
& x_{n_{j}}^{+}-x_{n_{i}}^{+} \leq 0, \quad 0 \leq i<j \leq k, \quad n=1, \ldots, N, \\
& x_{n_{k}}-x_{n_{k}}^{+} \leq 0, \quad n=1, \ldots, N, \\
& x_{n_{i}}{ }^{-} \geq 0, x_{n_{i}}^{+} \geq 0, \quad i=0,1, \ldots, k, \quad n=1, \ldots, N .
\end{aligned}
$$

Regarding $\tilde{z} \in \mathcal{F}_{C}$, the objective of the (FFLP), as a multi-objective function $f$ : $\mathbb{R}^{2(k+1) \times N} \rightarrow \mathbb{R}^{2(k+1)}$, which argument $x$ is defined by the parameters of the $N k$-polygonal fuzzy variables $x=\left(x_{1}, \ldots, x_{N}\right) \in \mathbb{R}^{2(k+1) \times N}$, with $x_{n}=\left(x_{n_{0}}^{-}, x_{n_{1}}^{-}, \ldots, x_{n_{k}}^{-}, x_{n_{k}}^{+}, \ldots\right.$, $\left.x_{n_{1}}^{+}, x_{n_{0}}^{+}\right)$, for $n=1, \ldots, N$.

The linear functions of its image $f(x)=\left(f_{0}^{-}(x), f_{1}^{-}(x), \ldots, f_{k}^{-}(x), f_{k}^{+}(x), \ldots, f_{1}^{+}(x)\right.$, $\left.f_{0}^{+}(x)\right)$ are defined as the sum of the $N$ coefficients (18),

$$
f_{i}^{-}(x)=\sum_{n=1}^{N}\left(\tilde{c}_{n} \tilde{x}_{n}\right)_{i}^{-}, \quad f_{i}^{+}(x)=\sum_{n=1}^{N}\left(\tilde{c}_{n} \tilde{x}_{n}\right)_{i}^{+},
$$

for each $i=0, \ldots, k$. All the constraints, $2(k+1) \times M+(2 k+1) \times N$, are represented as linear inequalities on the variable $x$. Constraints (21) and (22) are the corresponding terms to (16), whereas constraints (23) to (25) correspond with the ordering of the fuzzy variables $\tilde{x}_{n}$.

Then, (MOLP) is a multiobjective linear programming problem. In this point, let us recall that a feasible point $x^{*} \in \mathbb{R}^{2(k+1) \times N}$ of (MOLP) is said to be a weakly efficient solution for the Minimization problem (MOLP) if there does not exist another feasible point $x$ such that $f\left(x^{*}\right)<f(x)$, that is, $f_{i}^{-}\left(x^{*}\right)<f_{i}^{-}(x)$ and $f_{i}^{+}\left(x^{*}\right)<f_{i}^{+}(x)$ for $i=0,1, \ldots, k$. The same for the Maximization problem, but replacing $>$ by $<$ in the previous expression.

The relationship between the (FFLP) and (MOLP) solutions is demonstrated in the following theorem.

Theorem $2 \tilde{x}=\left(\tilde{x}_{1}, \ldots, \tilde{x}_{N}\right)$, with $\tilde{x}_{n}=\left(x_{n_{0}}^{-}, x_{n}^{-}, \ldots, x_{n_{k}}^{-}, x_{n_{k}}^{+}, \ldots, x_{n_{1}}^{+}, x_{n_{0}}^{+}\right) \in \mathcal{F}_{C}$, $n=1, \ldots, N$, is a nondominated solution of (FFLP) if and only if $x=\left(x_{1_{0}}^{-}, x_{1_{1}}^{-}, \ldots\right.$, $\left.x_{1}^{-}, x_{1}^{+}, \ldots, x_{1}^{+}, x_{1}^{+}, \ldots, x_{N_{0}^{-}}^{-}, x_{N_{1}}^{-}, \ldots, x_{N_{k}^{-}}^{-}, x_{N_{k}^{+}}^{+}, \ldots, x_{N_{1}^{+}}^{+}, x_{N_{0}^{+}}^{+}\right) \in \mathbb{R}^{2(k+1) \times N}$ is a weakly efficient solution of (MOLP).

Proof Let us consider a minimization process for (FFLP) and for (MOLP), and recall that all variables and coefficients in (FFLP) are $k$-polygonal fuzzy numbers with respect to $\left\{0=\alpha_{0}<\alpha_{0}<\cdots<\alpha_{k}=1\right\}$, a partition of the [0,1] interval. Let us prove that $x=\left(x_{10}^{-}, x_{1}^{-}, \ldots, x_{1_{k}^{-}}^{-}, x_{1}^{+}, \ldots, x_{1}^{+}, x_{1}^{+}, \ldots, x_{N_{0}^{-}}^{-}, x_{N_{1}}^{-}, \ldots, x_{N_{k}}^{-}, x_{N_{k}}^{+}, \ldots, x_{N_{1}^{+}}^{+}\right.$, $\left.x_{N_{0}^{+}}^{+}\right) \in \mathbb{R}^{2(k+1) \times N}$ is a feasible solution for (MOLP) if and only if $\tilde{x}=\left(\tilde{x}_{1}, \ldots, \tilde{x}_{N}\right)$, with $\tilde{x}_{n}=\left(x_{n_{0}}^{-}, x_{n}^{-}, \ldots, x_{n_{k}}^{-}, x_{n_{k}}^{+}, \ldots, x_{n}{ }_{1}^{+}, x_{n_{0}}^{+}\right) \in \mathcal{F}_{C}, n=1, \ldots, N$ is a feasible solution for (FFLP). To this purpose, if $x=\left(x_{1}^{-}, x_{1}^{-}, \ldots, x_{1}^{-}, x_{1}^{+}, \ldots, x_{1}^{+}, x_{1}^{+}, \ldots, x_{N_{0}}^{-}\right.$, $\left.x_{N_{1}}^{-}, \ldots, x_{N_{k}}^{-}, x_{N_{k}}^{+}, \ldots, x_{N_{1}^{+}}^{+}, x_{N_{0}^{+}}^{+}\right) \in \mathbb{R}^{2(k+1) \times N}$ is a feasible solution for (MOLP), then the conditions 


$$
x_{n_{i}}^{-}-x_{n}{ }_{j}^{-} \leq 0, \quad x_{n_{j}}^{+}-x_{n_{i}}^{+} \leq 0, \quad x_{n_{k}}^{-}-x_{n_{k}}^{+} \leq 0, \quad x_{n_{i}}^{-} \geq 0,
$$

are held for all $0 \leq i<j \leq k, n=1, \ldots, N$. These previous conditions are equivalent to state that $\tilde{x}_{n}$ is a nonnegative $k$-polygonal fuzzy number, for all $n=1, \ldots, N$. Furthermore, by the direct application of the definition of $\leqq$, it follows that the remaining feasibility conditions on $x$ in (MOLP), given by (21) and (22) are equivalent to the feasibility conditions (16) and (17) on $\tilde{x}=\left(\tilde{x}_{1}, \ldots, \tilde{x}_{n}\right)$ in (FFLP). Therefore, it is derived that $x$ is a feasible solution for (MOLP) if and only if $\tilde{x}$ is a feasible solution for (FFLP).

Now, let us suppose that $x$ is a weakly efficient solution of (MOLP), and, following, we prove that the feasible solution $\tilde{x}$ is a nondominated solution of (FFLP). To this aim, suppose the contrary, that is, there exists a feasible solution $\tilde{y}=\left(\tilde{y}_{1}, \ldots, \tilde{y}_{N}\right)$ for (FFLP), with $\tilde{y}_{n}=\left(y_{n_{0}}^{-}, y_{n}{ }_{1}^{-}, \ldots, y_{n_{k}}^{-}, y_{n_{k}}^{+}, \ldots, y_{n_{1}}^{+}, y_{n_{0}}^{+}\right) \in \mathcal{F}_{C}, n=1, \ldots, N$, such that

$$
\sum_{n=1}^{N} \tilde{c}_{n} \tilde{y}_{n} \prec \sum_{n=1}^{N} \tilde{c}_{n} \tilde{x}_{n}
$$

Condition (27) is equivalent to

$$
\sum_{n=1}^{N}\left(\tilde{c}_{n} \tilde{y}_{n}\right)_{i}^{-}<\sum_{n=1}^{N}\left(\tilde{c}_{n} \tilde{x}_{n}\right)_{i}^{-}, \quad \sum_{n=1}^{N}\left(\tilde{c}_{n} \tilde{y}_{n}\right)_{i}^{+}<\sum_{n=1}^{N}\left(\tilde{c}_{n} \tilde{x}_{n}\right)_{i}^{+},
$$

for each $i=0, \ldots, k$. Since $\tilde{y}$ is feasible for (FFLP), it follows that $y=\left(y_{10}^{-}, y_{1}^{-}, \ldots\right.$, $\left.y_{1}^{-}, y_{1}^{+}, \ldots, y_{1}^{+}, y_{1}^{+}, \ldots, y_{N_{0}^{-}}^{-}, y_{N_{1}^{-}}^{-}, \ldots, y_{N_{k}^{-}}^{-}, y_{N_{k}^{+}}^{+}, \ldots, y_{N_{1}^{+}}^{+}, y_{N_{0}^{+}}^{+}\right) \in \mathbb{R}^{2(k+1) \times N}$ is a feasible solution for (MOLP). By (28), we have that $x$ is not a weakly efficient solution of (MOLP), what is a contradiction. Therefore, it follows that if $x$ is a weakly efficient solution of (MOLP), then $\tilde{x}$ is a nondominated solution of (FFLP). Conversely, in a similar manner as before, let us suppose that $\tilde{x}$ is a nondominated solution of (FFLP), and let us prove that $x$ is a weakly efficient solution of (MOLP). Since $\tilde{x}$ is feasible for (FFLP), it follows that $x$ is feasible for (MOLP). Let us suppose that $x$ is not a weakly efficient solution of (MOLP). This means that there exists a feasible solution $y=\left(y_{1_{0}}^{-}, y_{1_{1}}^{-}, \ldots, y_{1_{k}}^{-}, y_{1_{k}}^{+}, \ldots, y_{1}^{+}\right.$, $\left.y_{1}^{+}, \ldots, y_{N_{0}}^{-}, y_{N_{1}}^{-}, \ldots, y_{N_{k}}^{-}, y_{N_{k}^{+}}^{+}, \ldots, y_{N_{1}^{+}}^{+}, y_{N_{0}^{+}}^{+}\right) \in \mathbb{R}^{2(k+1) \times N}$ for (MOLP) such that $f_{i}^{-}(y)<f_{i}^{-}(x)$ and $f_{i}^{+}(y)<f_{i}^{+}(x)$ for $i=0,1, \ldots, k$, what implies (28), with $\tilde{y}=\left(\tilde{y}_{1}, \ldots, \tilde{y}_{N}\right)$ for (FFLP). And (28) is equivalent to (27), which means that $\tilde{x}$ is a nondominated solution of (FFLP), what is a contradiction with our initial assumptions. Therefore, it is proved that if $\tilde{x}$ is a nondominated solution of (FFLP), then $x$ is a weakly efficient solution of (MOLP). The proof of the result under maximization process for (FFLP) and for (MOLP) is similar to the previous one. In consequence, the proof is complete.

\section{Algorithm to generate the nondominated solutions set for (FFLP)}

\subsection{A method to generate a subset of nondominated solutions of (FFLP)}

There exist several methods to generate weakly efficient solutions of (MOLP). One of them is by means of related weighted sum problems (see [2,4]). A formulation of this type of optimization problem can be as follows. Given (MOLP) and $w=\left(w_{1}, \ldots, w_{2(k+1)}\right) \in$ $\mathbb{R}^{2(k+1)}, w_{i} \geq 0, \sum_{i=1}^{2(k+1)} w_{i}=1$, we define the related weighted sum problem as 


$$
\begin{aligned}
(\operatorname{MOLP})_{w} & \operatorname{Min} / \operatorname{Max} \sum_{i=1}^{2(k+1)} w_{i} f_{i}(x)=w_{1} \sum_{n=1}^{N}\left(\tilde{c}_{n} \tilde{x}_{n}\right)_{0}^{-}+\ldots+w_{2(k+1)} \sum_{n=1}^{N}\left(\tilde{c}_{n} \tilde{x}_{n}\right)_{0}^{+} \\
\text {subject to }(21)--(26) & (29)
\end{aligned}
$$

Theorem 3 Given $w=\left(w_{1}, \ldots, w_{2(k+1)}\right) \in \mathbb{R}^{2(k+1)}, w_{i} \geq 0, \sum_{i=1}^{2(k+1)} w_{i}=1$, if $x \in$ $\mathbb{R}^{2(k+1) \times N}$ is an optimal solution of the weighted optimization problem $(M O L P)_{w}$, then $x$ is a weakly efficient solution of (MOLP).

As a consequence of the previous result, we have the following one to determine non dominated solutions of the (FFLP).

Theorem 4 Given $w=\left(w_{1}, \ldots, w_{2(k+1)}\right) \in \mathbb{R}^{2(k+1)}, w_{i} \geq 0, \sum_{i=1}^{2(k+1)} w_{i}=1$, if $x=\left(x_{1_{0}}^{-}\right.$, $x_{1}^{-}, \ldots, x_{1}^{-}, x_{1}^{+}, \ldots, x_{1}^{+}, x_{1}^{+}, \ldots, x_{N_{0}^{-}}^{-}, x_{N_{1}}^{-}, \ldots, x_{N_{k}}^{-}, x_{N_{k}}^{+}, \ldots, x_{N_{1}^{+}}^{+}$, $\left.x_{N_{0}^{+}}^{+}\right) \in \mathbb{R}^{2(k+1) \times N}$ is an optimal solution of the weighted optimization problem $(M O L P)_{w}$, then $\tilde{x}=\left(\tilde{x}_{1}, \ldots, \tilde{x}_{N}\right)$, with $\hat{x}_{n}=\left(x_{n_{0}}^{-}, x_{n}{ }_{1}^{-}, \ldots, x_{n_{k}}^{-}, x_{n_{k}}^{+}, \ldots, x_{n_{1}}^{+}, x_{n_{0}}^{+}\right) \in \mathcal{F}_{C}, n=$ $1, \ldots, N$, is a non dominated solution of (FFLP).

Proof If $x=\left(x_{10}^{-}, x_{1}^{-}, \ldots, x_{1}^{-}, x_{1}^{+}, \ldots, x_{1}^{+}, x_{1}^{+}, \ldots, x_{N_{0}}^{-}, x_{N_{1}}^{-}, \ldots, x_{N_{k}^{-}}^{-}, x_{N_{k}}^{+}\right.$, $\left.\ldots, x_{N}^{+}, x_{N_{0}^{+}}^{+}\right) \in \mathbb{R}^{2(k+1) \times N}$ is an optimal solution of the weighting optimization problem $(\text { MOLP })_{w}$, then, by Theorem 3, it follows that $x$ is a weakly efficient solution of (MOLP). Then, by Theorem 2 , we have that $\tilde{x}=\left(\tilde{x}_{1}, \ldots, \tilde{x}_{N}\right)$, with $\tilde{x}_{n}=\left(x_{n_{0}}^{-}, x_{n_{1}}^{-}, \ldots, x_{n_{k}}^{-}, x_{n_{k}}^{+}\right.$, $\ldots, x_{n_{1}}^{+}, x_{n_{0}}^{+}$) for $n=1, \ldots, N$, is a nondominated solution of (FFLP).

Theorem 4 provides a simple way to get non dominated solutions for (FFLP). So a first approach for generating non dominated solutions can be made via the search of such weights, which leads to an optimal solution of the associated weighted sum problem of the (MOLP). The outline of the method is shown in the Algorithm 1, which generates a single non dominated solution of the (FFLP) at each run.

Theorem 5 If we run Algorithm 1 and get $S_{(F F L P)} \neq \emptyset$, then any $\tilde{x} \in S_{(F F L P)}$ is a nondominated solution of (FFLP).

Proof The proof is inmediate from Theorem 4.

\subsection{A method to construct and generate the whole set of nondominated solutions of (FFLP)}

The previous algorithm determines each weight $w$ randomly, at each run. Despite the possibility of an empty subset of non dominated solutions output from the Algorithm 1, this can be negligible for a total number of iterations big enough. The next natural step is determining the whole set of non dominated solutions of the (FFLP) problem, or its generating set, besides refining the weights selection for the associated weighted sum problem (MOLP) $)_{w}$.

The new approach presented at the current work of the (FFLP) problems is not only the generalization from triangular fuzzy numbers type to more general $k$-polygonal fuzzy numbers, defined in (1). Besides, the development of an algorithm for getting the weakly efficient solutions set of the associated (MOLP), and therefore for (FFLP) problem itself. Moreover, it characterizes the structure of the weakly efficient solutions set by means of a generating set. 


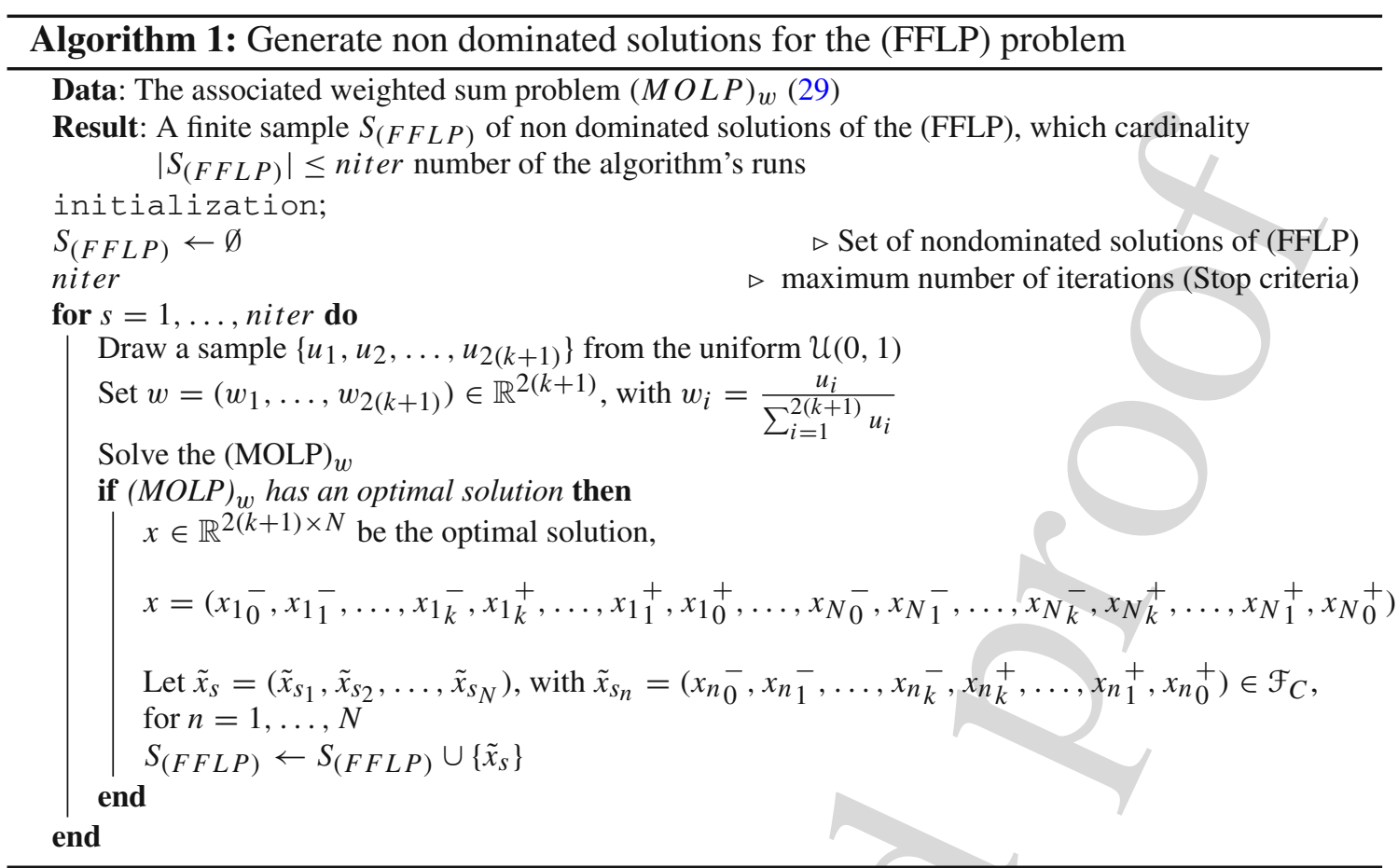

As it was described in the previous section, Theorem 2 establishes the relation between (FFLP) and (MOLP) non dominated solutions. This is, we can determine the weakly efficient solutions set of (FFLP) problem, (15)--(17), by solving the corresponding multi-objective linear programming (MOLP) problem, (20)--(26). A general and well-known method for generating weakly efficient solutions is to solve its associated weighted sum problem, for a given weight of $w$, as the Algorithm 1 outlines.

Our method is based on the algorithm proposed by [20], for obtaining all weak efficient solutions in a multi-objective linear programming (MOLP) problem. Yan et al. [46] originally developed it, but based on the assumption of a finite optimal solution for all the weighted sum problems to be solved throughout the algorithm development. Foroughi and Jafari [20] improved this methodology, including the unbounded cases as well.

Its main potential is to generate the solution set, if it exists, just solving some weighted sum problems. It calculates the corresponding weights $w_{i}$ for $i=1, \ldots, r$ during the process and provides a clear and easy solution structure of the solution set as well.

A combination of Theorems 4 and 5 from [46], which demonstrate the above assertions, adapted to the current notation in a matrix way is the following.

Theorem 6 Given a multi objective linear programming,

$$
(M O L P) \min / \max F(x)=C x
$$

$$
\begin{array}{ll}
\text { s.t. } & A x=b \\
& x \geq 0
\end{array}
$$

The weakly efficient solutions set, $R_{w p}$, of the $(M O L P)$ problem is obtained from a finite number of weighted sum problems,

$$
\begin{aligned}
(M O L P)_{w_{j}} \min / \max & w_{j}^{T} C x \\
\text { s.t. } & A x=b \\
& x \geq 0
\end{aligned}
$$


given $w_{j} \geq 0$, for $j=1, \ldots, J$. So it can be represented as $R_{w p}=\bigcup_{j=1}^{J} R_{w p}^{j}$, where $R_{w p}^{j}=\left\{x: A x=b, \quad w_{j}^{T} C x=\alpha_{j}, x \geq 0\right\}$, and the pairs $\left(w_{j}, \alpha_{j}\right)^{T}$ are computed at each iteration of the Algorithm 2 as the extreme rays of the polyhedral cone

$$
S=\left\{\left(\begin{array}{l}
F \\
\alpha
\end{array}\right) \mid \begin{array}{r}
\left(C x^{i}\right)^{T} F \geq \alpha, x^{i} \in R \\
\left(C d^{i}\right)^{T} F \geq 0, d^{i} \in D \\
F \geq 0, F \neq 0
\end{array}\right\},
$$

defined by the set of extreme optimal solutions of the $(M O L P)_{w_{j}}$ problems gathered up to that iteration, denoted by $R=\left\{x^{1}, x^{2}, \ldots\right\}$, and the extreme directions for unbounded cases, $D=\left\{d^{1}, d^{2}, \ldots\right\}$.

Proof After writing the above problems (20)-(26) in their standard format, the result is immediate applying the corresponding theorems from [46] and [20]. As well as the calculation of the weights $\left(w_{k}, \alpha_{k}\right)^{T}$, for $k=1, \ldots, K$.

\section{Numerical examples}

In this section, we show the application of the proposed new method to find the fuzzy optimal (non dominated) solutions of FFLP problems with inequality constraints, through two illustrative but straightforward problems.

Algorithms 1 and 2, which determine the whole set of the non dominated solutions for (FFLP) problem through a finite generator set, have been implemented in $\mathrm{R}^{1}$ (version 3.3.2), and making use of the lpsolve package for solving Linear Programs. The codes are run on an Intel Core i7 macOS 10.14.3, 2.2 GHz, 8 GB RAM, 1600 MHz DDR3.

\subsection{Example 1}

Let consider the following small example of a fully fuzzy linear programming (FFLP) problem, very similar to one proposed in [3]. To show not only the proposed FFLP modelization but also how both Algorithms 1 and 2 run.

Due to the small number of variables, not only the Algorithm 2 is pretty fast, but it also makes possible the computation of all the extreme points of each subset $R_{w p}^{j}$ within a reasonable computational consumption. Therefore, both the whole structure of the weakly efficient solutions set, $R_{w p}$ (30), as well as its sum-form expression (31) are obtained without a high computational time consumption (2.1257 min in total).

$($ FFLP1 $) \operatorname{Min} \tilde{z}=(-1,0,2) \tilde{x}_{1}+(1,2,3) \tilde{x}_{2} \in \mathcal{F}_{C}$

$$
\begin{aligned}
\text { s.t. } \quad(2,5,8) \tilde{x}_{1}+(3,4,10) \tilde{x}_{2} \leqq(1,3,6) \\
(4,5,7) \tilde{x}_{1}+(0,5,15) \tilde{x}_{2} \leqq(2,3,6)
\end{aligned}
$$$$
\tilde{x}_{1}, \tilde{x}_{2} \geqq 0
$$

There are two fuzzy variables, and two constraints with triangular fuzzy numbers, this is a 1-polygonal case. Following the multiobjective linear problem (MOLP) associated is

\footnotetext{
1 https://www.r-project.org. $\mathrm{R}$ is a language and environment for statistical computing and graphics. It is a GNU project which is similar to the $\mathrm{S}$ language and environment which was developed at Bell Laboratories (formerly AT\&T, now Lucent Technologies) by John Chambers and colleagues.
} 


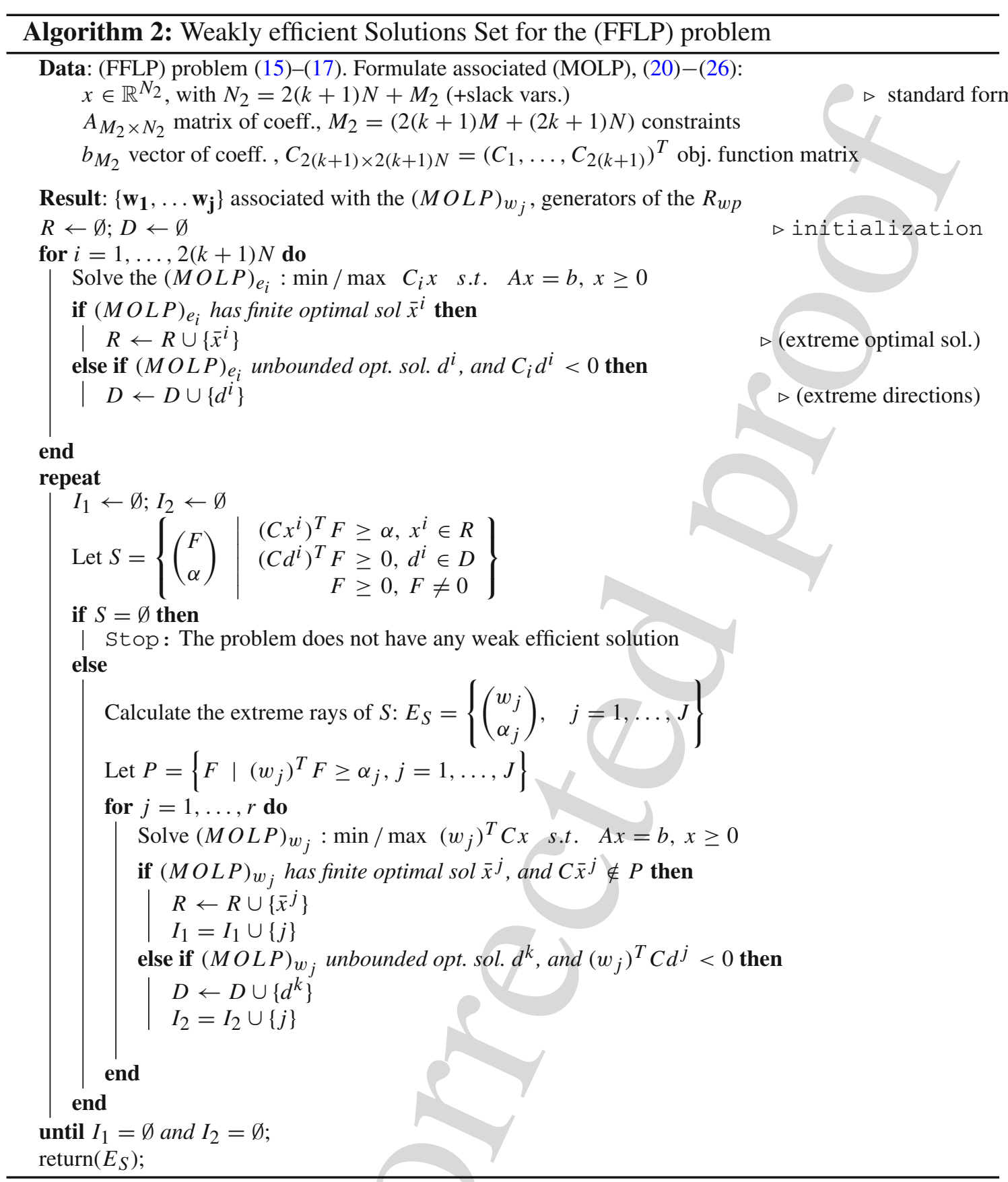

formulated, where the multiobjective function $f=\left(f_{1}, f_{2}, f_{3}\right): \mathbb{R}^{6} \rightarrow \mathbb{R}^{3}$ is a vectorvalued function, evaluated on $x=\left(x_{1}^{-}, \hat{x}_{1}, x_{1}^{+}, x_{2}^{-}, \hat{x}_{2}, x_{2}^{+}\right) \in \mathbb{R}^{6}$, with

$$
f_{1}(x)=-x_{1}^{+}+x_{2}^{-}, f_{2}(x)=2 \hat{x}_{2} \text { and } f_{3}(x)=2 x_{1}^{+}+3 x_{2}^{+} .
$$

Note $c_{10}^{-}=-1 \leq 0$, then $z_{1}^{-}=\left(\tilde{c}_{1} \tilde{x}_{1}\right)^{-}=\min \left\{c_{1}^{-} x_{1}^{-}, c_{1}^{-} x_{1}^{+}, c_{1}^{+} x_{1}^{-}, c_{1}^{+} x_{1}^{+}\right\}=c_{1}^{-} x_{1}^{+}$ $=-x_{1}^{+}$, and $f_{1}(x)=-x_{1}^{+}+x_{2}^{-}$(see (18) and Remark 3$)$.

Regarding the simplicity in notation, for the particular triangular fuzzy numbers case, it is applied the equivalent notation $\tilde{x}_{n}=\left(x_{n_{0}}^{-}, x_{n_{1}}^{-}, x_{n_{1}}^{+}, x_{n_{0}}^{+}\right) \equiv\left(x_{n}^{-}, \hat{x}_{n}, x_{n}^{+}\right)$, since $x_{n_{1}}^{-}=x_{n_{1}}^{+}$ for $n=1,2$. 
$\left(\right.$ MOLP1) Min $f(x)=\left(-x_{1}^{+}+x_{2}^{-}, 2 \hat{x}_{2}, 2 x_{1}^{+}+3 x_{2}^{+}\right) \in \mathbb{R}^{3}$

s.t.

$$
\begin{aligned}
2 x_{1}^{-}+3 x_{2}^{-} & \leq 1, \\
5 \hat{x}_{1}+4 \hat{x}_{2} & \leq 3, \\
8 x_{1}^{+}+10 x_{2}^{+} & \leq 6, \\
4 x_{1}^{-} & \leq 2, \\
5 \hat{x}_{1}+5 \hat{x}_{2} & \leq 3, \\
7 x_{1}^{+}+15 x_{2}^{+} & \leq 6, \\
x_{n}^{-}-\hat{x}_{n} & \leq 0, \quad n=1,2 \\
\hat{x}_{n}-x_{n}^{+} & \leq 0, \quad n=1,2 \\
x_{n}^{-}, \hat{x}_{n}, x_{n}^{+} & \geq 0, \quad n=1,2
\end{aligned}
$$

There are $M_{2}=3 M+3 N=10$ constraints, and $3 N=6$ variables. Equivalently, the above problem in matrix notation results as

(MOLP1) Min $C x \in \mathbb{R}^{3}$

$$
\text { s.t. } A x \leq b, \quad x \geq 0
$$

where,

$$
C=\left(\begin{array}{cccccc}
0 & 0 & -1 & 1 & 0 & 0 \\
0 & 0 & 0 & 0 & 2 & 0 \\
0 & 0 & 2 & 0 & 0 & 3
\end{array}\right), \quad A=\left(\begin{array}{ccccccc}
2 & 0 & 0 & 3 & 0 & 0 \\
0 & 5 & 0 & 0 & 4 & 0 \\
0 & 0 & 8 & 0 & 0 & 10 \\
4 & 0 & 0 & 0 & 0 & 0 \\
0 & 5 & 0 & 0 & 5 & 0 \\
0 & 0 & 7 & 0 & 0 & 15 \\
1 & -1 & 0 & 0 & 0 & 0 \\
0 & 1 & -1 & 0 & 0 & 0 \\
0 & 0 & 0 & 1 & -1 & 0 \\
0 & 0 & 0 & 0 & 1 & -1
\end{array}\right),
$$

$b=(1,3,6,2,3,6,0,0,0,0)^{T}$, and $x=\left(x_{1}^{-}, \hat{x}_{1}, x_{1}^{+}, x_{2}^{-}, \hat{x}_{2}, x_{2}^{+}\right)^{T} \in \mathbb{R}^{6}$. For Algorithm 2 application, (MOLP1) problem is rewritten in standard form, adding the slacks variables $s_{i}$ for $i=1, \ldots, M_{2}$. This is,

(MOLP1) Min $C^{\prime} x^{\prime} \in \mathbb{R}^{3}$

$$
\text { s.t. } A^{\prime} x^{\prime}=b, \quad x^{\prime} \geq 0
$$

$C^{\prime}=\left[\begin{array}{ll}C & 0_{3 \times 10}\end{array}\right], A^{\prime}=\left[\begin{array}{ll}A & I_{10 \times 10}\end{array}\right]$, and $x^{\prime}=\left(x_{1}^{-}, \hat{x}_{1}, x_{1}^{+}, x_{2}^{-}, \hat{x}_{2}, x_{2}^{+}, s_{1}, \ldots, s_{10}\right)^{T} \in \mathbb{R}^{16}$.

Step 1 (Initialization) For $i=1,2,3$, we solve the weighted linear programs

$$
\begin{aligned}
(M O L P 1)_{1}: & \operatorname{Min} f_{1}\left(x^{\prime}\right)=-x_{1}^{+}+x_{2}^{-} \\
& \text {s.t. } A^{\prime} x^{\prime}=b, x^{\prime} \geq 0 \\
(M O L P 1)_{2}: & \text { Min } f_{2}\left(x^{\prime}\right)=2 \hat{x}_{2} \\
& \text { s.t. } A^{\prime} x^{\prime}=b, x^{\prime} \geq 0 \\
(M O L P 1)_{3}: & \text { Min } f_{3}\left(x^{\prime}\right)=2 x_{1}^{+}+3 x_{2}^{+} \\
& \text {s.t. } A^{\prime} x^{\prime}=b, \quad x^{\prime} \geq 0
\end{aligned}
$$

$(\text { MOLP } 1)_{i}$ have bounded optimal solutions: $x^{\prime 1}=(1 / 2,3 / 5,3 / 4,0,0,0,0,0$, $0,0,0,3 / 4,1 / 10,3 / 20,0,0)^{T}$, with objective value $C^{\prime} x^{\prime 1}=(-3 / 4,0,3 / 2)^{T}$; $x^{\prime 2}=(1 / 2,3 / 5,3 / 5,0,0,3 / 25,0,0,0,0,0,0,1 / 10,0,0,3 / 25)^{T}$, with 
$C^{\prime} x^{\prime 2}=(-3 / 5,0,39 / 25)^{T}$; and $x^{\prime 3}=(0,0,0,0,0,0,1,3,6,2,3,6,0$, $0,0,0)^{T}$, with $C^{\prime} x^{\prime 3}=(0,0,0)^{T}$, respectively. Let denote $R=\left\{x^{\prime 1}, x^{\prime 2}, x^{\prime 3}\right\}$ and $D=\emptyset$, the initial sets of optimal solutions and extreme directions (if unbounded feasible region, which is not the case).

Step 2 (First iteration of the algorithm). Calculate the first weights $\mathbf{w}_{\mathbf{j}}$ associated with the weighted sum problems $(M O L P)_{w_{j}}$, generators of the weakly efficient solutions set of (MOLP1). Let $I_{1}=\emptyset$, and $I_{2}=\emptyset$. And denote,

$$
S^{1}=\left\{\left(\begin{array}{l}
f_{1} \\
f_{2} \\
f_{3} \\
\alpha
\end{array}\right):\left(\begin{array}{ccc}
-3 / 4 & 0 & 3 / 2 \\
-3 / 5 & 0 & 39 / 25 \\
0 & 0 & 0
\end{array}\right)\left(\begin{array}{l}
f_{1} \\
f_{2} \\
f_{3}
\end{array}\right) \geq\left(\begin{array}{c}
\alpha \\
\alpha \\
\alpha
\end{array}\right),\left(\begin{array}{l}
f_{1} \\
f_{2} \\
f_{3}
\end{array}\right)>0\right\}
$$

All extreme rays of the polyhedral cone $S^{1}$ are $E_{S}=\left\{(0,1,0,0)^{T},(2 / 3,0,1 / 3\right.$, $\left.0)^{T},(4 / 7,0,0,-3 / 7)^{T},(0,0,1,0)^{T}\right\}$, so

$$
P^{1}=\left\{\left(\begin{array}{l}
f_{1} \\
f_{2} \\
f_{3}
\end{array}\right):\left(\begin{array}{ccc}
0 & 1 & 0 \\
2 / 3 & 0 & 1 / 3 \\
4 / 7 & 0 & 0 \\
0 & 0 & 1
\end{array}\right)\left(\begin{array}{l}
f_{1} \\
f_{2} \\
f_{3}
\end{array}\right) \geq\left(\begin{array}{c}
0 \\
0 \\
-3 / 7
\end{array}\right)\right\}
$$

Step 3 We get four new weights, $w_{1}=(0,1,0)^{T}, w_{2}=(2 / 3,0,1 / 3)^{T}, w_{3}=(4 / 7,0,0)^{T}$, and $w_{4}=(0,0,1)^{T}$. We use them to get new weak efficient solutions, for $j=1,2,3,4$ :

$$
\begin{aligned}
(\operatorname{MOLP} 1)_{j}: & \operatorname{Min}\left(w_{j}\right)^{T} C^{\prime} x^{\prime} \\
\text { s.t. } & A^{\prime} x^{\prime}=b, \quad x^{\prime} \geq 0
\end{aligned}
$$

but the image in the objective space of their optimal solutions are all in $P^{1}$, i.e., $C^{\prime} x^{\prime j} \in P^{1}$. Therefore, $R^{1}$ remains the same, as well as the index sets $I_{1}$ and $I_{2}$.

Step 4 Note that, if both $I_{1}=\emptyset$ and $I_{2}=\emptyset$, the algorithm stops. Otherwise, repeat from Step 2.

The weakly efficient solutions set of the problem is defined as

$$
R_{w p}=\bigcup_{j=1}^{4} R_{w p}^{j}=\bigcup_{j=1}^{4}\left\{x: A x \leq b,\left(w_{j}\right)^{T} C x=\alpha_{j}, x \geq 0\right\}
$$

where the pairs of $\left(\begin{array}{l}w_{j} \\ \alpha_{j}\end{array}\right)$ have been computed by the Algorithm 2, based on Theorem 6 .

$\left.E_{S}=\left\{\left(\begin{array}{l}w_{1} \\ \alpha_{1}\end{array}\right)=\left(\left(\begin{array}{c}0 \\ 1 \\ 0\end{array}\right),\left(\begin{array}{l}w_{2} \\ \alpha_{2}\end{array}\right)=\left(\left(\begin{array}{c}2 / 3 \\ 0 \\ 1 / 3\end{array}\right)\right),\left(\begin{array}{l}w_{3} \\ \alpha_{3}\end{array}\right)=\left(\begin{array}{c}4 / 7 \\ 0 \\ 0\end{array}\right)\right),\left(\begin{array}{l}w_{4} \\ \alpha_{4}\end{array}\right)=\left(\begin{array}{c}0 \\ 0 \\ -3 / 7\end{array}\right)\right)\right\}$

These weights (not normalized) are shown in Table 1, along with the extreme points of the $R_{w p}^{j}$ polyhedrons, which computation has been possible due to the small size of the problem. And allows us to represent the above polyhedral subsets $R_{w p}^{j}$ in their sum-form, given by the convex combination of their extreme points. Moreover, it is clear to see that $R_{w p}^{2} \subseteq R_{w p}^{1}$, $R_{w p}^{3} \subseteq R_{w p}^{1}$, and $R_{w p}^{4} \subseteq R_{w p}^{1}$. Hence, the weakly efficient solutions set of (MOLP1), and therefore the nondominated solutions set of (FFLP1) problem, can be simply expressed as 
Table 1 Output of the Algorithm 2, this is the set of weights (not normalized) and $\alpha$ which determine the set of all weak efficient solutions of the (MOLP1) problem, by means of the subsets $R_{w p}^{j}$

\begin{tabular}{|c|c|c|c|}
\hline$j$ & $w_{j} \in \mathbb{R}^{3}$ & $\alpha_{j}$ & $\begin{array}{l}\text { Extreme points of } R_{w p}^{j}=\left\{x: A x \leq b,\left(w_{j}\right)^{T} C x\right. \\
\left.=\alpha_{j}, x \geq 0\right\} x=\left(x_{1}^{-}, \hat{x}_{1}, x_{1}^{+}, x_{2}^{-}, \hat{x}_{2}, x_{2}^{+}\right)\end{array}$ \\
\hline 1 & $(0,1,0)$ & 0 & $\begin{array}{l}u^{1}=\left(\frac{1}{2}, \frac{1}{2}, \frac{1}{2}, 0,0,0\right), u^{2}=\left(\frac{1}{2}, \frac{1}{2}, \frac{1}{2}, 0,0, \frac{1}{6}\right), u^{3}=\left(\frac{1}{2}, \frac{3}{5}, \frac{3}{5}, 0,0,0\right), \\
u^{4}=\left(\frac{1}{2}, \frac{3}{5}, \frac{3}{5}, 0,0, \frac{3}{25}\right), u^{5}=\left(\frac{1}{2}, \frac{1}{2}, \frac{3}{4}, 0,0,0\right), u^{6}=\left(\frac{1}{2}, \frac{1}{2}, \frac{3}{5}, 0,0, \frac{3}{25}\right), \\
u^{7}=\left(\frac{1}{2}, \frac{3}{5}, \frac{3}{4}, 0,0,0\right), u^{8}=(0,0,0,0,0,0), u^{9}=\left(0,0,0,0,0, \frac{2}{5}\right), \\
u^{10}=\left(0,0, \frac{3}{4}, 0,0,0\right), u^{11}=\left(0,0, \frac{3}{5}, 0,0, \frac{3}{25}\right), u^{12}=\left(0, \frac{3}{5}, \frac{3}{5}, 0,0,0\right), \\
u^{13}=\left(0, \frac{3}{5}, \frac{3}{5}, 0,0, \frac{3}{25}\right), u^{14}=\left(0, \frac{3}{5}, \frac{3}{4}, 0,0,0\right)\end{array}$ \\
\hline 2 & $(2,0,1)$ & 0 & $u^{1}, u^{3}, u^{5}, u^{7}, u^{8}, u^{10}, u^{12}, u^{13}$ \\
\hline 3 & $(4,0,0)$ & -3 & $u^{5}, u^{7}, u^{10}, u^{14}$ \\
\hline 4 & $(0,0,1)$ & 0 & $u^{8}$ \\
\hline
\end{tabular}

Last column shows the extreme points of these subsets $R_{w p}^{j}$, for its sum-form representation

$$
R_{w p}=R_{w p}^{1}=\left\{\sum_{i=1}^{14} \lambda_{i} u^{i}: e^{T} \lambda=1, \lambda \geq 0, \lambda \in \mathbb{R}^{14}\right\}
$$

In this way, we can generate any weak efficient solution of the (FFLP) problem. For example, for $\lambda=(1,0, \ldots, 0) \in \mathbb{R}^{14}$ we have the weakly efficient solution $x=\left(x_{1}^{-}\right.$, $\left.\hat{x}_{1}, x_{1}^{+}, x_{2}^{-}, \hat{x}_{2}, x_{2}^{+}\right)=u^{1}=(1 / 2,1 / 2,1 / 2,0,0,0)$ of the (MOLP1). The corresponding nondominated solution of the (FFLP1) problem is $\tilde{x}_{1}=\left(x_{1}^{-}, \hat{x}_{1}, x_{1}^{+}\right)=(1 / 2,1 / 2,1 / 2)$, and $\tilde{x}_{2}=\left(x_{2}^{-}, \hat{x}_{2}, x_{2}^{+}\right)=(0,0,0)$, with $\tilde{z}=(0,0,0)$.

This is a great step forward in comparison with Algorithm 1, since we can determine all the weak efficient solutions. In fact, after 1000 runs of Algorithm 1 (using random weights at each iteration) we only get two different solutions, $x=(0,0,0,0,0,0)=u^{8}$, and $x=(0,0,3 / 4,0,0,0)=u^{10}$. The results, for comparison purposes, are shown in Table 2.

As commented in Sect. 1, in this manner, a decision-maker gets the whole set of fuzzy optimal solutions, to manage them at their convenience or suitability. Although it is beyond the scope of this paper, we give some options to give a precise quantity for each fuzzy variable or solution, through the defuzzification to scalars (see Ross [41]).

The simplest method is the Max membership principle or height method, this is just taking as defuzzified value $(\tilde{a})^{M}=x^{*}$ the corresponding to the $\alpha=1$ level. I.e., $\tilde{a}\left(x^{*}\right) \geq \tilde{a}(x)$, for all $x \in\left[a_{0}^{-}, a_{0}^{+}\right]$. In the particular case of triangular fuzzy numbers, it corresponds to the central value $\hat{a}$.

Another procedure is to compute the center of area or center of gravity, $(\tilde{a})^{C}$ $\int x \tilde{a}(x) d x / \int \tilde{a}(x) d x$, known as the Centroid method. Both methods have been computed for the weak efficient solutions for the (FFLP1) problem given at the last columns of Table 2.

Finally, although one can consider that all triangular fuzzy numbers have the same shape, one can find a variety of the graphs of their membership functions, as shown in Fig. 3. 


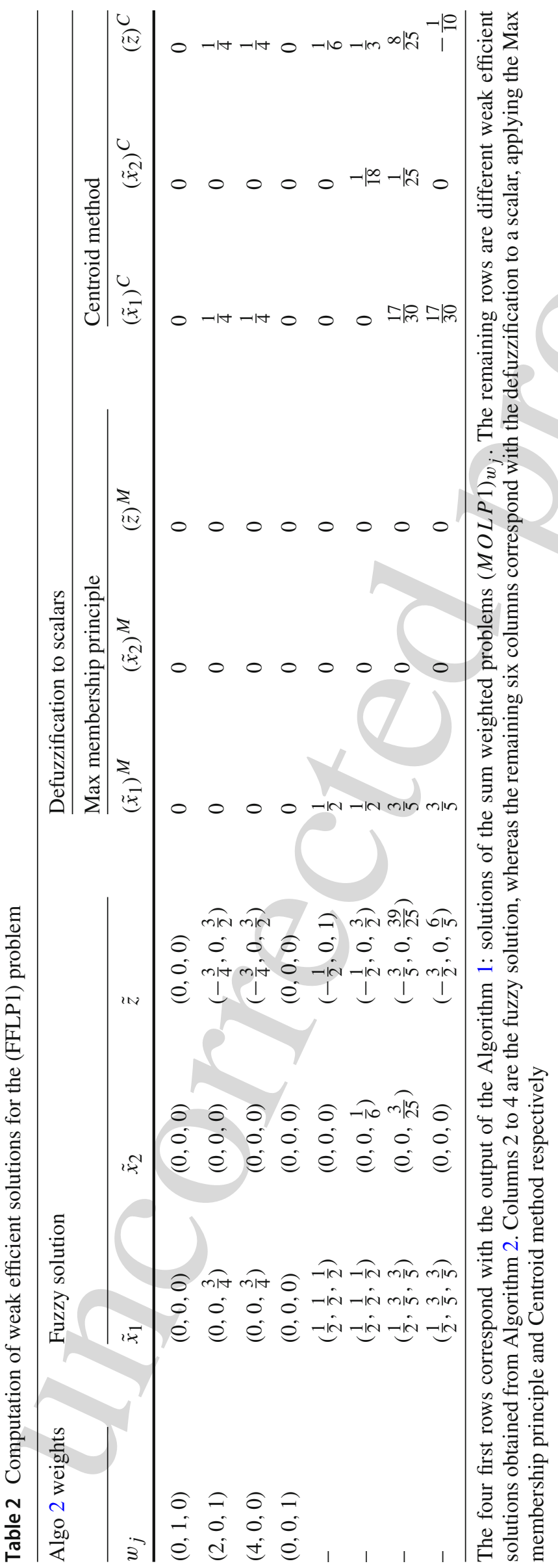



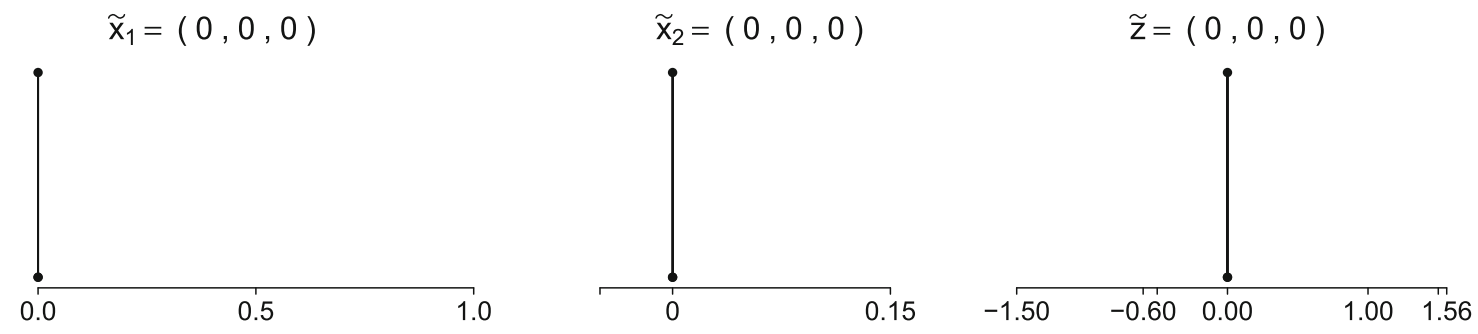

$\widetilde{x}_{1}=(0,0,3 / 4)$

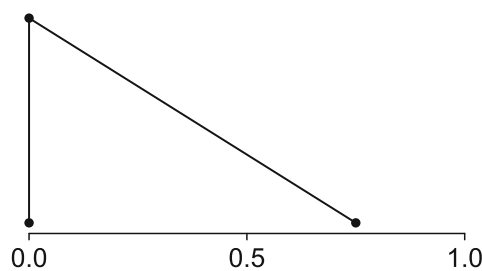

$\widetilde{\mathrm{x}}_{2}=(0,0,0)$

$\widetilde{z}=(-3 / 2,0,3 / 2)$

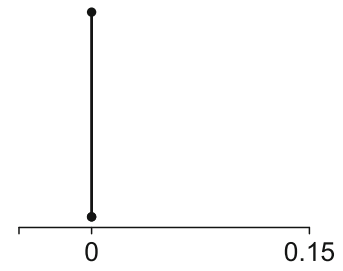

$\widetilde{x}_{1}=(1 / 2,1 / 2,1 / 2)$

$\widetilde{x}_{2}=(0,0,0)$
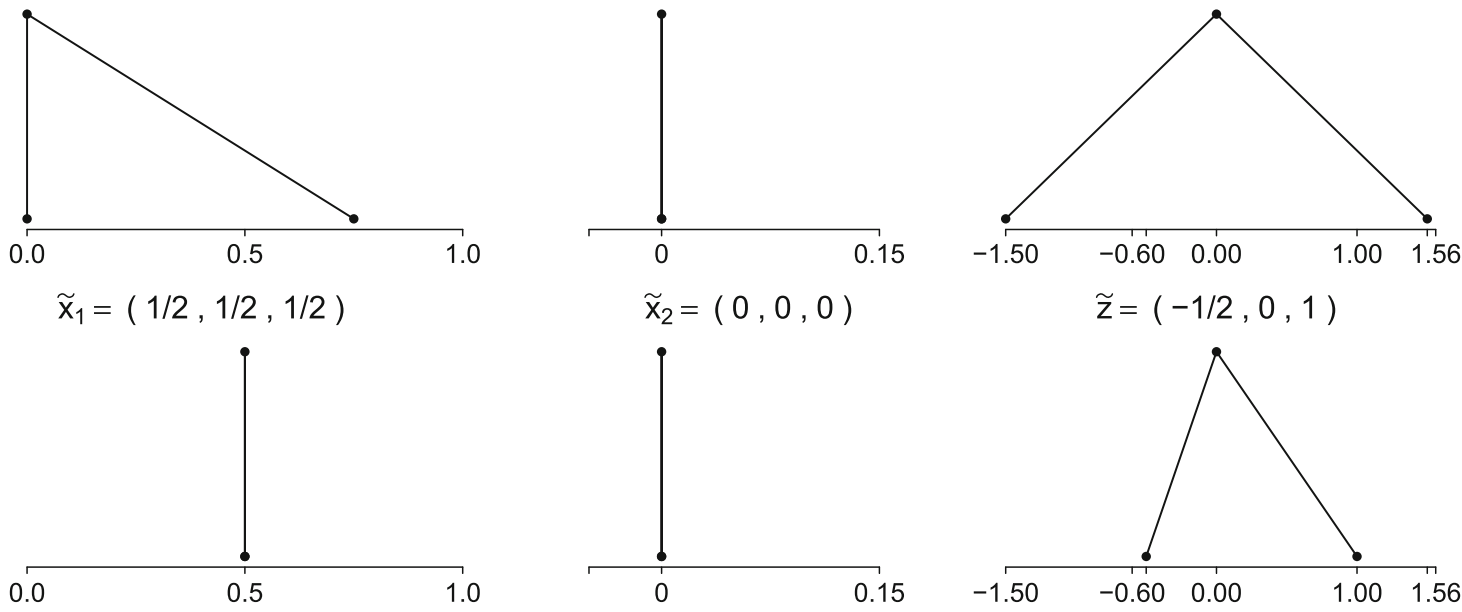

$\widetilde{x}_{1}=(1 / 2,3 / 5,3 / 5)$

$\widetilde{\mathrm{x}}_{2}=(0,0,3 / 25)$
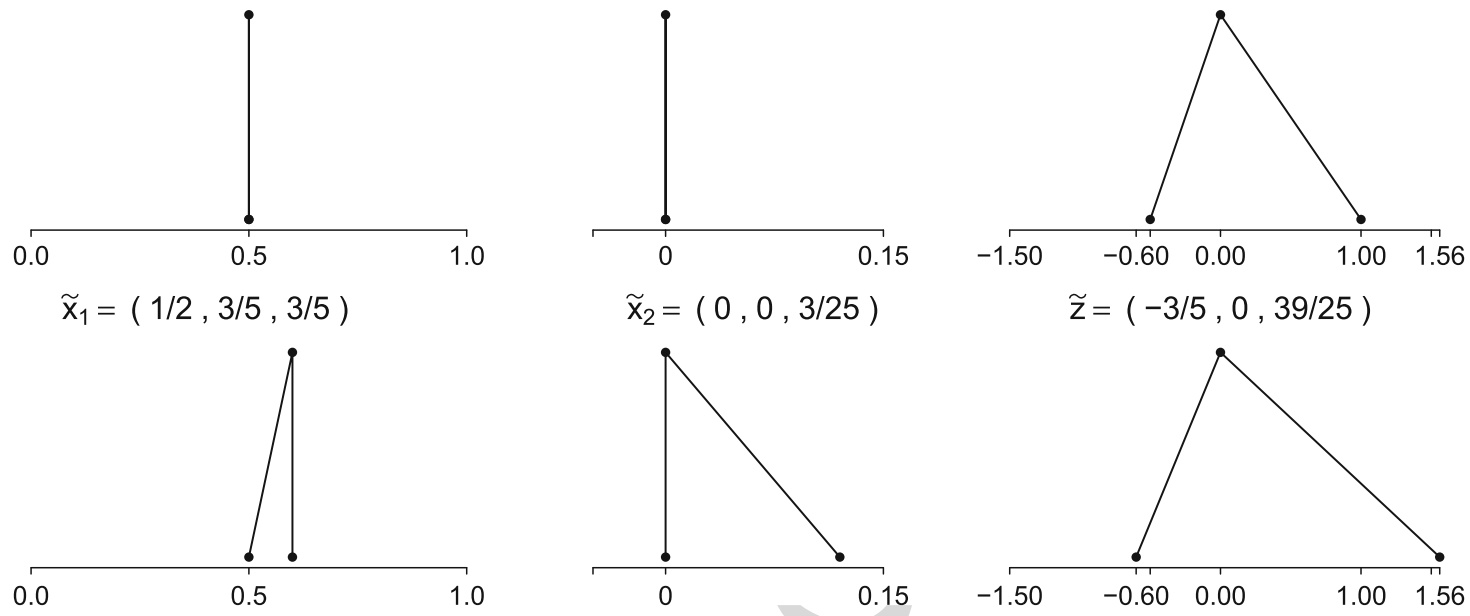

Fig. 3 Some weak efficient solutions for the (FFLP1) problem, from Example 1, computed through its corresponding (MOLP1) and the Algorithms 1 and 2. See Table 2

\subsection{Example 2}

Consider now the next example, for 2-polygonal fuzzy numbers.

(FFLP2) Min $\tilde{z}=\left(-1,-\frac{1}{2}, 0, \frac{1}{2}, 1\right) \tilde{x}_{1}+(1,3,5,7,9) \tilde{x}_{2} \in \mathcal{F}_{C}$

s.t. $(2,3,5,6,8) \tilde{x}_{1}+\left(3, \frac{7}{2}, 4,6,10\right) \tilde{x}_{2} \leqq(1,2,3,5,6)$

$(3,4,5,6,7) \tilde{x}_{1}+(0,3,5,9,15) \tilde{x}_{2} \leqq(1,2,3,4,6)$

$\tilde{x}_{1}, \tilde{x}_{2} \geqq 0$

The particular case of pentagonal fuzzy numbers as a 2-polygonal fuzzy number, $\tilde{x}_{n}$ $=\left(x_{n_{0}}^{-}, x_{n_{1}}^{-}, x_{n_{2}}^{-}, x_{n_{2}}^{+}, x_{n_{1}}^{+}, x_{n_{0}}^{+}\right)$, where $x_{n_{2}}^{-}=x_{n_{2}}^{+}=\hat{x}_{n}$ for $n=1,2$. The corresponding multi objective programming problem (MOLP), with $x=\left(x_{10}^{-}, x_{1}^{-}, x_{12}, x_{1}^{+}, x_{1_{0}}^{+}, x_{2}^{-}\right.$, $\left.x_{21}^{-}, x_{22}, x_{2}^{+}, x_{2}^{+}\right) \in \mathbb{R}^{10}$, is 
$\left(\right.$ MOLP2) Min $f(x)=\left(-x_{10}^{-}+x_{2}^{-},-\frac{1}{2} x_{1}^{-}+3 x_{2}^{-}, 5 x_{22}, \frac{1}{2} x_{1}^{+}+7 x_{2}^{+}, x_{10}^{+}+9 x_{2}^{+}\right) \in \mathbb{R}^{5}$ s.t.

$$
\begin{aligned}
& 2 x_{1}^{-}+3 x_{20}^{-} \leq 1, \\
& 3 x_{1}^{-}+\frac{7}{2} x_{2}^{-} \leq 2 \text {, } \\
& 5 x_{12}+4 x_{22} \leq 3 \text {, } \\
& 6 x_{1}{ }_{1}^{+}+6 x_{2}{ }_{1}^{+} \leq 5 \text {, } \\
& 8 x_{10}^{+}+10 x_{20}^{+} \leq 6 \text {, } \\
& 3 x_{10}^{-} \leq 1, \\
& 4 x_{1}{ }_{1}^{-}+3 x_{2}{ }_{1}^{-} \leq 2 \text {, } \\
& 5 x_{12}+5 x_{22} \leq 3 \text {, } \\
& 6 x_{1}^{+}+9 x_{2}^{+} \leq 4, \\
& 7 x_{10}^{+}+15 x_{2}^{+} \leq 6 \text {, } \\
& x_{n_{0}}^{-}-x_{n_{1}}^{-} \leq 0, \quad n=1,2 \\
& x_{n_{1}}^{-}-x_{n_{2}} \leq 0, \quad n=1,2 \\
& x_{n_{2}}-x_{n_{1}}^{+} \leq 0, \quad n=1,2 \\
& x_{n_{1}}^{+}-x_{n_{0}}^{+} \leq 0, \quad n=1,2 \\
& x_{n_{0}}^{-}, x_{n_{1}}^{-}, x_{n_{2}}, x_{n_{1}}^{+}, x_{n_{0}}^{+} \geq 0, \quad n=1,2
\end{aligned}
$$

Or equivalently, in matrix notation,

(MOLP2) Min $C x \in \mathbb{R}^{5}$

$$
\text { s.t. } A x \leq b, \quad x \geq 0
$$

where,

$$
C=\left(\begin{array}{cccccccccc}
-1 & 0 & 0 & 0 & 0 & 1 & 0 & 0 & 0 & 0 \\
0 & -\frac{1}{2} & 0 & 0 & 0 & 0 & 3 & 0 & 0 & 0 \\
0 & 0 & 0 & 0 & 0 & 0 & 0 & 5 & 0 & 0 \\
0 & 0 & 0 & \frac{1}{2} & 0 & 0 & 0 & 0 & 7 & 0 \\
0 & 0 & 0 & 0 & 1 & 0 & 0 & 0 & 0 & 9
\end{array}\right)
$$




$$
A=\left(\begin{array}{cccccccccc}
2 & 0 & 0 & 0 & 0 & 3 & 0 & 0 & 0 & 0 \\
0 & 3 & 0 & 0 & 0 & 0 & 7 & 0 & 0 & 0 \\
0 & 0 & 5 & 0 & 0 & 0 & 0 & 4 & 0 & 0 \\
0 & 0 & 0 & 6 & 0 & 0 & 0 & 0 & 6 & 0 \\
0 & 0 & 0 & 0 & 8 & 0 & 0 & 0 & 0 & 10 \\
3 & 0 & 0 & 0 & 0 & 0 & 0 & 0 & 0 & 0 \\
0 & 4 & 0 & 0 & 0 & 0 & 3 & 0 & 0 & 0 \\
0 & 0 & 5 & 0 & 0 & 0 & 0 & 5 & 0 & 0 \\
0 & 0 & 0 & 6 & 0 & 0 & 0 & 0 & 9 & 0 \\
0 & 0 & 0 & 0 & 7 & 0 & 0 & 0 & 0 & 15 \\
1 & -1 & 0 & 0 & 0 & 0 & 0 & 0 & 0 & 0 \\
0 & 1 & -1 & 0 & 0 & 0 & 0 & 0 & 0 & 0 \\
0 & 0 & 1 & -1 & 0 & 0 & 0 & 0 & 0 & 0 \\
0 & 0 & 0 & 1 & -1 & 0 & 0 & 0 & 0 & 0 \\
0 & 0 & 0 & 0 & 0 & 1 & -1 & 0 & 0 & 0 \\
0 & 0 & 0 & 0 & 0 & 0 & 1 & -1 & 0 & 0 \\
0 & 0 & 0 & 0 & 0 & 0 & 0 & 1 & -1 & 0 \\
0 & 0 & 0 & 0 & 0 & 0 & 0 & 0 & 1 & -1
\end{array}\right) \text { and } b=\left(\begin{array}{l}
1 \\
2 \\
3 \\
5 \\
6 \\
1 \\
2 \\
3 \\
4 \\
6 \\
0 \\
0 \\
0 \\
0 \\
0 \\
0 \\
0 \\
0
\end{array}\right)
$$

A simple problem of $N=2$ fully fuzzy variables, parametrized as $k=2$-polygonal fuzzy numbers (pentagonal, $2(k+1)-1=2 k+1=5$, since $x_{j_{2}}^{-}=x_{j_{2}}^{+}=x_{j_{2}}$ ), and $M=2$ constraints becomes a multi objective problem with $2 k+1=5$ objective functions, $(2 k+1) N=10$ variables, and $(2 k+1) M+(2 k) N=18$ constraints. The Algorithm 2 is still pretty fast for such size, and easy to implement. It only took $20.95899^{\prime \prime}$ to compute the set of weights $\left(w_{j}, \alpha_{j}\right)$, see Table 3 .

The set of all weakly efficient solutions of the (MOLP2) problem is defined as,

$$
R_{w p}=\bigcup_{j=1}^{9}\left\{x: A x \leq b,\left(w_{j}\right)^{T} C x=\alpha_{j}, x \geq 0\right\}
$$

Just the increase in the parametrization applied to the previous example, from triangular to pentagonal fuzzy numbers, makes the calculation of all the extreme points of the corresponding polyhedral subsets $R_{w p}^{j}$ a harsh computational problem, which is not the aim of the present work. There are no extreme directions, since the feasible region is bounded. So, we only apply the Algorithm 1 to get the set of finite weights $w_{j}$, which define the $R_{w p}^{j}$ subsets, and the whole structure of all the weakly efficient solutions of the (MOLP).

As it is shown in Table 3, this first algorithm based on the solutions given by the sumweighted (MOLP) problems is limited to find only a few different weak efficient solutions. Whereas the Algorithm 2 is able to establish the whole set of all weak efficient solutions, it is computationally constrained by the size of the final (MOLP) problem. As in the previous Example, Sect. 5.1, the last columns of Table 3 are the defuzzification to scalars of the optimal fuzzy solutions, computed with the Centroid method. The Max membership principle procedure has not been included since, for all the solutions shown in the table, they are just the central value $(\tilde{x})^{M}=x_{2}^{-}=x_{2}^{+}=0$.

Once more, one can find a variety in the 2-polygonal fuzzy numbers shape, as shown in Fig. 4. 


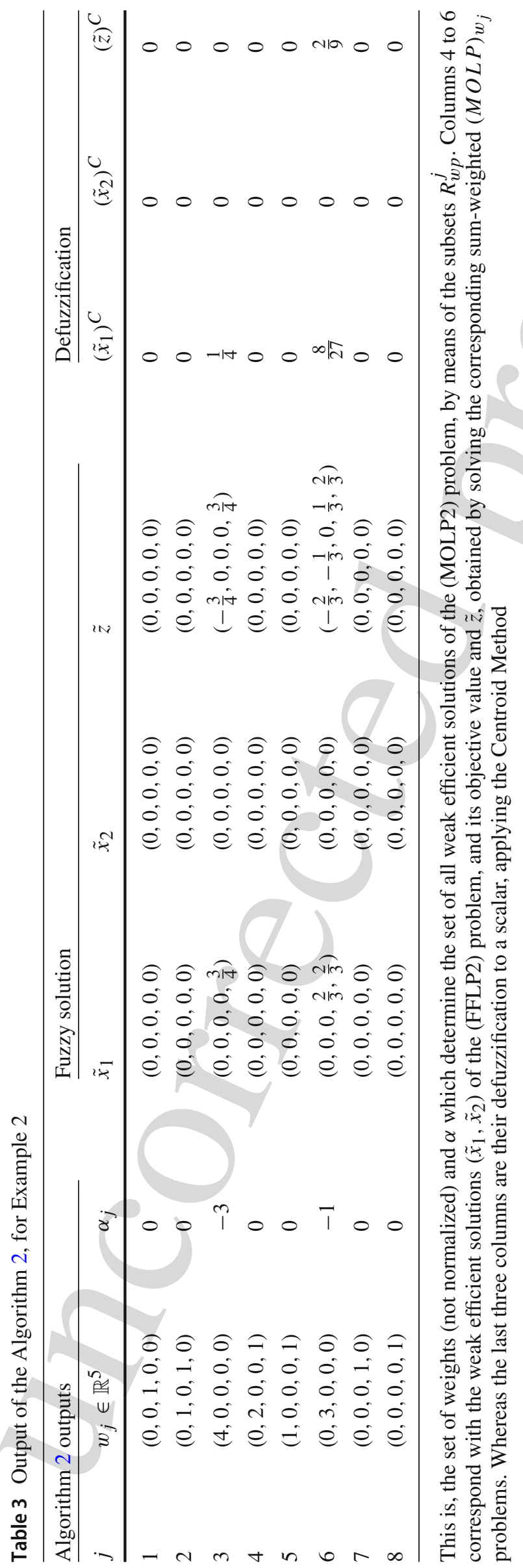




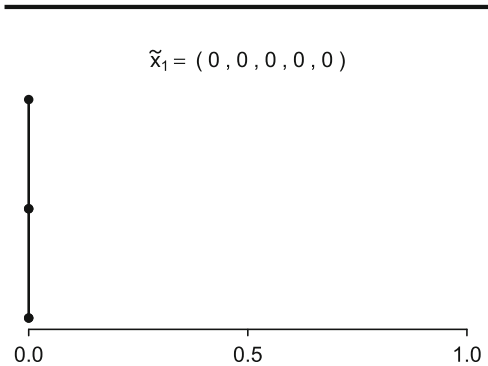

$\tilde{x}_{1}=(0,0,0,0,3 / 4)$

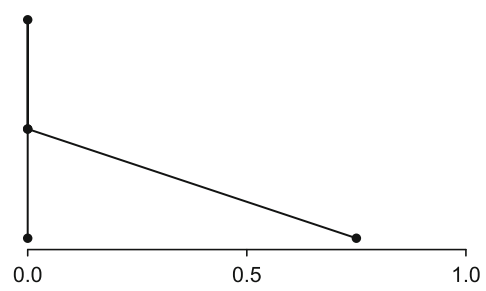

$\tilde{\mathrm{x}}_{1}=(0,0,0,2 / 3,2 / 3)$

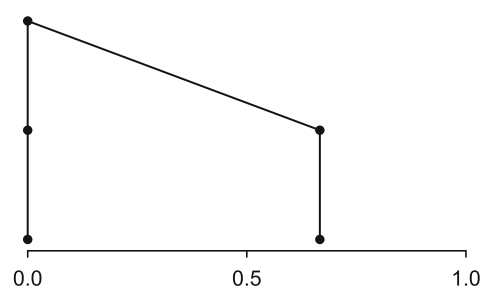

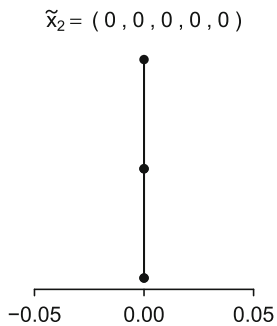

$\tilde{\mathrm{x}}_{2}=(0,0,0,0,0)$

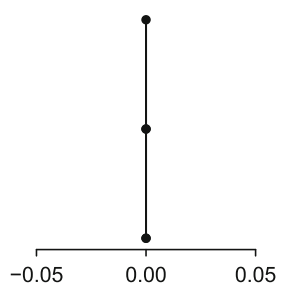

$\tilde{\mathrm{x}}_{2}=(0,0,0,0,0)$

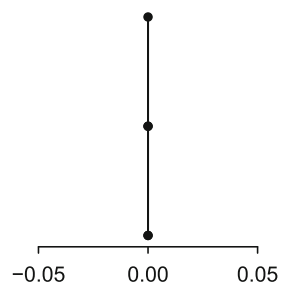

$\widetilde{\mathbf{z}}=(0,0,0,0,0)$

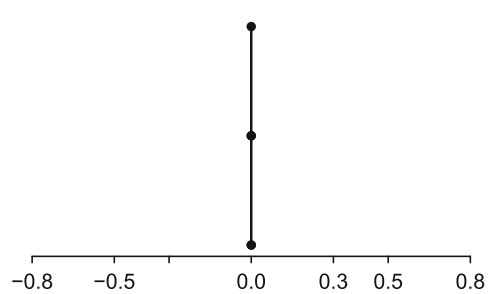

$\tilde{\mathbf{z}}=(-3 / 4,0,0,0,3 / 4)$

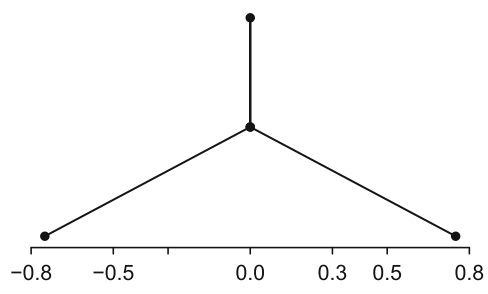

$\tilde{z}=(-2 / 3,-1 / 3,0,1 / 3,2 / 3)$

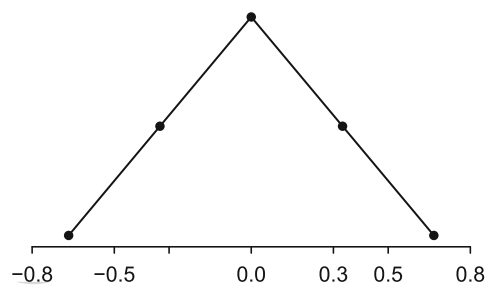

Fig. 4 Some weak efficient solutions for the (FFLP2) problem, from Example 2, computed through its corresponding (MOLP2) and the Algorithms 1 and 2. See Table 3

\section{Conclusions}

This work addresses how to solve fully fuzzy linear programming problems (FFLP), with fuzzy numbers parameterized as $k$-polygonal ones, through its counterpart multiobjective linear programming problem (MOLP). In this regard, a fully fuzzy problem (FFLP) links to a multiobjective crisp linear problem without any information loss, usually not avoided when this transformation is made via ranking functions.

Furthermore, the whole set of nondominated solutions of the (FFLP) is obtained by solving a finite number of linear, crisp programming problems.

Acknowledgements The first author was partially supported by the research project MTM2017-89577P (MINECO, Spain), and the second author was partially supported by Spanish Ministry of Economy and Competitiveness through grants AYA2016-75931-C2-1-P, AYA2015-68012-C2-1, AYA2014-57490-P, AYA2013-40611-P, and from the Consejería de Educación y Ciencia (Junta de Andalucía) through TIC-101, TIC-4075 and TIC-114.

\section{References}

1. Alefeld, G., Herzberger, J.: Introduction to Interval Computations. Academic Press, New York (1983)

2. Arana-Jiménez, M. (ed.): Optimiality Conditions in Vector Optimization. Bentham Science Publishers Ltd, Bussum (2010)

3. Arana-Jiménez, M., Rufián-Lizana, A., Chalco-Cano, Y., Román-Flores, H.: Generalized convexity in fuzzy vector optimization through a linear ordering. Inf. Sci. 312, 13-24 (2015)

4. Arana-Jiménez, M., Antczak, T.: The minimal criterion for the equivalence between local and global optimal solutions in nondifferentiable optimization problem. Math. Methods Appl. Sci. 1-9 (2017) 
5. Arana-Jiménez, M.: Nondominated solutions in a fully fuzzy linear programming problem. Math. Methods Appl. Sci. 41, 7421-7430 (2018)

6. Báez-Sáncheza, A.D., Morettib, A.C., Rojas-Medar, M.A.: On polygonal fuzzy sets and numbers. Fuzzy Sets Syst. 209, 54-65 (2012)

7. Bellman, R.E., Zadeh, L.A.: Decision making in a fuzzy environment. Manag. Sci. 17, 141-164 (1970)

8. Bector, C.R., Chandra, S.: Fuzzy Mathematical Programming and Fuzzy Matrix Games. Springer, Berlin (2005)

9. Bhardwaj, B., Kumar, A.: A note on the paper "A simplified novel technique for solving fully fuzzy linear programming problems”. J. Optim. Theory Appl. 163, 685-696 (2014)

10. Campos, L., Verdegay, J.L.: Linear programming problems and ranking of fuzzy numbers. Fuzzy Set. Syst. 32, 1-11 (1989)

11. Chakraborty, D., Jana, D.K., Roy, T.K.: A new approach to solve fully fuzzy transportation problem using triangular fuzzy number. Inte. J. Oper. Res. 26, 153-179 (2016)

12. Chen, S.-M., Adam, S.I.: Weighted fuzzy interpolated reasoning based on ranking values of polygonal fuzzy sets and new scale and move transformation techniques. Inf. Sci. 435, 184-202 (2018)

13. Coroianu, L., Gagolewski, M., Grzegorzewski, P.: Piecewise linear approximation of fuzzy numbers: algorithms, arithmetic operations and stability of characteristics. Soft. Comput. (2019). https://doi.org/ 10.1007/s00500-019-03800-2

14. Das, S.K., Mandal, T., Edalatpanah, S.A.: A mathematical model for solving fully fuzzy linear programming problem with trapezoidal fuzzy numbers. Appl. Intell. 46(3), 509-519 (2017)

15. Das, S.K., Edalatpanah, S.A., Mandal, T.: A proposed model for solving fuzzy linear fractional programming problem: numerical point of view. J. Comput. Sci. 25, 367-375 (2018)

16. Dubois, D., Prade, H.: Operations on fuzzy numbers. Int. J. Syst. Sci. 9, 613-626 (1978)

17. Dubois, D., Prade, H.: Fuzzy Sets and Systems: Theory and Applications. Academic Press, New York (1980)

18. Ebrahimnejad, A., Nasseri, S.H., Lotfi, F.H., Soltanifar, M.: A primal-dual method for linear programming problems with fuzzy variables. Eur. J. Ind. Eng. 4, 189-209 (2010)

19. Ezzati, R., Khorram, E., Enayati, R.: A new algorithm to solve fully fuzzy linear programming problems using the MOLP problem. Appl. Math. Model. 39, 3183-3193 (2015)

20. Foroughi, A.A., Jafari, Y.: A modified method for constructing efficientsolutions structure of MOLP. Appl. Math. Model. 33, 2403-2410 (2009)

21. Ganesan, K., Veeramani, P.: Fuzzy linear programs with trapezoidal fuzzy numbers. Ann. Oper. Res. 143, 305-315 (2006)

22. Ghaznavi, M., Soleimani, F., Hoseinpoor, N.: Parametric analysis in fuzzy number linear programming problems. Int. J. Fuzzy Syst. 18(3), 463-477 (2016)

23. Goestschel, R., Voxman, W.: Elementary fuzzy calculus. Fuzzy Sets Syst. 18, 31-43 (1986)

24. Guerra, M.L., Stefanini, L.: A comparison index for interval based on generalized Hukuhara difference. Soft. Comput. 16, 1931-1943 (2012)

25. Hanss, M.: Applied Fuzzy Arithmetic. Springer, Stuttgart (2005)

26. Kaufmann, A., Gupta, M.M.: Introduction to Fuzzy Arithmetic Theory and Applications. Van Nostrand Reinhold, New York (1985)

27. Khan, I.U., Ahmad, T., Maan, N.: A simplified novel technique for solving fully fuzzy linear programming problems. J. Optim. Theory Appl. 159, 536-546 (2013)

28. Khan, I.U., Ahmad, T., Maan, N.: A reply to a note on the paper "A simplified novel technique for solving fully fuzzy linear programming problems". J. Optim. Theory Appl. 173, 353-356 (2017)

29. Kovářová, L., Viertl, R.: The generation of fuzzy sets and the constructions of characterizing functions of fuzzy data. Iran. J. Fuzzy Syst. 12(6), 1-16 (2015)

30. Kumar, A., Kaur, J., Singh, P.: A new method for solving fully fuzzy linear programming problems. Appl. Math. Model. 35, 817-823 (2011)

31. Liu, B.: Uncertainty Theory. Springer, Berlin (2015)

32. Liu, Q., Gao, X.: Fully fuzzy linear programming problem with triangular fuzzy numbers. J. Comput. Theor. Nanosci. 13, 4036-4041 (2016)

33. Lotfi, F.H., Allahviranloo, T., Jondabeha, M.A., Alizadeh, L.: Solving a fully fuzzy linear programming using lexicography method and fuzzy approximate solution. Appl. Math. Modell. 33, 3151-3156 (2009)

34. Maleki, H.R., Tata, M., Mashinchi, M.: Linear programming with fuzzy variables. Fuzzy Set. Syst. 109, 21-33 (2000)

35. Maleki, H.R.: Ranking functions and their applications to fuzzy linear programming. Far East J. Math. Sci. 4, 283-301 (2002)

36. Möller, B., Beer, M., Liebscher, M.: Fuzzy analysis as alternative to stochastic methods-theoretical aspect. In: Proceedings of the 4th German LS-DYNA Forum 2005. Bamberg (2005) 
37. Moore, R.E.: Interval Analysis. Prentice-Hall, Englewood Cliffs (1966)

38. Moore, R.E.: Method and Applications of Interval Analysis. SIAM, Philadelphia (1979)

39. Najafi, H.S., Edalatpanah, S.A.: A note on "A new method for solving fully fuzzy linear programming problems". Appl. Math. Model. 37, 7865-7867 (2013)

40. Najafi, H.S., Edalatpanah, S.A., Dutta, H.: A nonlinear model for fully fuzzy linear programming with fully unrestricted variables and parameters. Alex. Eng. J. 55(3), 2589-2595 (2016)

41. Ross, T.J.: Fuzzy Logic with Engineering Applications. Wiley, New York (2010)

42. Stefanini, L., Arana-Jiménez, M.: Karush-Kuhn-Tucker conditions for interval and fuzzy optimization in several variables under total and directional generalized differentiability. Fuzzy Sets Syst. 362, 1-34 (2018)

43. Stefanini, L., Sorini, L., Guerra, M.L.: Parametric representation of fuzzy numbers and application to fuzzy calculus. Fuzzy Sets Syst. 157(18), 2423-2455 (2006)

44. Stefanini, L., Bede, B.: Generalized fuzzy differentiability with LU-parametric representation. Fuzzy Sets Syst. 257, 184-203 (2014)

45. Wang, G., Duan, Y.: TOPSIS approach for multi-attribute decision making problems based on nintuitionistic polygonal fuzzy sets description. Comput. Ind. Eng. 124, 573-581 (2018)

46. Yan, H., Wei, Q., Wang, J.: Constructing efficient solutions structure of multiobjective linear programming. J. Math. Anal. Appl. 307, 504-523 (2005)

47. Yasin Ali, M., Sultana, A., Khodadad Kha, A.F.M.: Comparison of fuzzy multiplication operation on triangular fuzzy number. IOSR J. Math. 12(4), 35-41 (2016)

Publisher's Note Springer Nature remains neutral with regard to jurisdictional claims in published maps and institutional affiliations. 